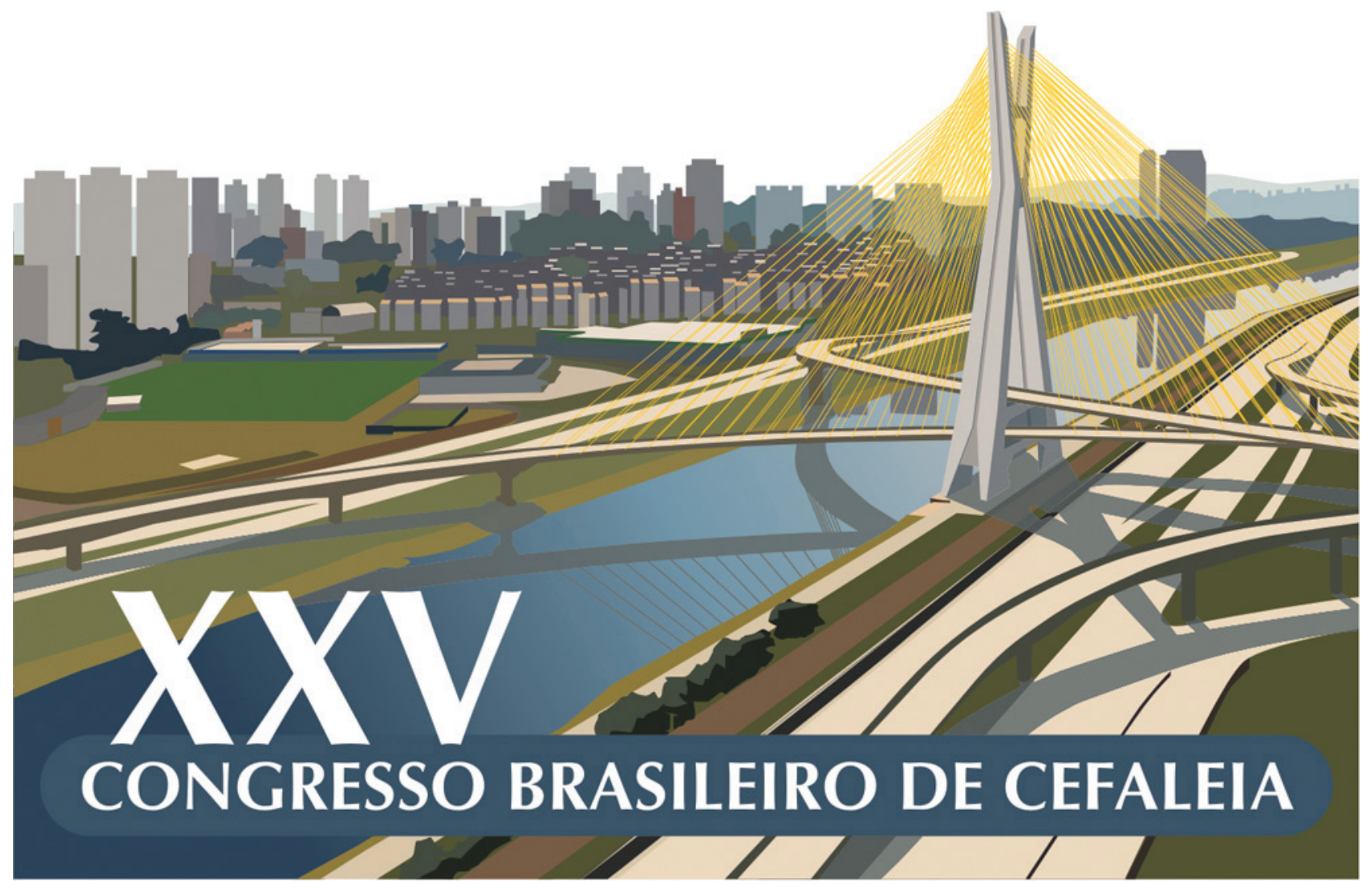

XXV Congresso Brasileiro de Cefaleia IHS Masters School

$1^{\circ}$ Brazilian-North American Synposium (BRANAS) VI Congresso do Comitê de Dor Orofacial

13 a 17 de setembro de 2011

São Paulo-SP

Programa Final 


\section{MENSAGEM DO PRESIDENTE}

Caro amigo,

Chegamos à $25^{a}$ edição do nosso tradicional Congresso Brasileiro de Cefaleia, a programação científica trouxe inúmeras novidades. Além dos nossos renomados palestrantes nacionais, contamos com a participação de dez professores internacionais que nos brindarão com palestras de alto nível. Esse ano realizaremos um encontro entre cefaliatras brasileiros e norte-americanos, o 1 st BRANAS (Brazilian - North American Headache Symposium) onde serão apresentados temas de alta complexidade pelos maiores expoentes dos dois países. A grade científica também procurou enfatizar a apresentação de casos clínicos através dos quais esperamos maior interatividade entres os palestrantes e a audiência. Serão discutidos aspectos clínicos, diagnóstico, terapêuticos e fisiopatológicos à luz dos casos apresentados, uma forma de estimular a participação de todos.

O VI Congresso de Dor Orofacial promete ser o melhor de todos os tempos, com uma programação muito bem elaborada pelo comitê científico e a presença das maiores autoridades nacionais. Estamos assistindo um crescente interesse por essa área nos nossos encontros, o qual está se tornando o principal evento brasileiro nesse campo da odontologia, pudemos comprovar esse interesse pelo número recorde de trabalhos enviados ao congresso.

Como é conhecimento de todos, nos dias 13 e 14 de setembro ocorre o Masters School da International Headache Society (MS-IHS), o primeiro curso desse gênero organizado pela IHS. Trata-se de uma imersão, dividida em duas etapas presenciais intercaladas por atividades online monitoradas pelos maiores especialistas mundiais, onde serão discutidos desde temas básicos até os assuntos mais profundos e atuais que cercam a ciência das cefaleias.

Esperamos que o $25^{\circ}$ Congresso Brasileiro de Cefaleia sirva de referência para suas próximas condutas.

Sejam bem-vindos!

$$
\text { Marcelo C Ciciarelli }
$$

Presidente da Sociedade Brasileira de Cefaleia 


\title{
COMISSÕES
}

\section{ORGANIZAÇÃO}

SBCe - Sociedade Brasileira de Cefaléia

\section{PRESIDENTES DO CONGRESSO}

Mario F. P. Peres e Celia Aparecida de Paula Roesler

\section{COMISSÃO ORGANIZADORA}

Marcelo C. Ciciarelli

Presidente

Luiz Paulo de Queiroz

Secretário

Carlos Alberto Bordini

Tesoureiro

\section{COMISSÃO CIENTÍFICA}

\author{
Carlos Alberto Bordini \\ Presidente \\ Jano Alves de Souza \\ Abouch Valenty Krymchantowski \\ Carla Jevoux \\ Mario F. P. Peres \\ Eliova Zukerman \\ José Geraldo Speciali \\ Pedro Ferreira Moreira Filho
}

\section{COMISSÃO DE PRÊMIOS E TRABALHOS}

\author{
José Geraldo Speciali \\ Coordenador \\ Carlos Alberto Bordini \\ Deusvenir de Souza Carvalho \\ Marcelo C. Ciciarelli \\ Marcelo Moraes Valença \\ Pedro Ferrereira Moreira Filho \\ Renan Barros Domingues \\ Renata Campi
}




\title{
PALESTRANTES
}

\section{PALESTRANTES ESTRANGEIROS}

\author{
Alan M. Rapoport - EUA \\ Hans Christoph Diener - Alemanha \\ Allan Purdy - Canadá \\ Michel Volcy Gomes - Colômbia \\ Benjamim Frishberg - EUA \\ Morris Levin - EUA \\ Robert Cowan - EUA \\ Robert Shapiro - EUA \\ Stephen D. Silberstein - EUA \\ Steve Baskin - EUA
}

\section{PALESTRANTES NACIONAIS}

\begin{abstract}
Alexandre Ottoni Kaup
Amelia Pasqual Marques

Ana Cristina Lotaif

Ana Luisa Lima Antoniazzi

Ana Maria Ladeira Yamada

Ariovaldo Alberto da Silva Junior

Carlos Alberto Bordini

Celia Aparecida de Paula Roesler

Cibele Dal Fabbro

Cinara Maria Camparis

Claudia Baptista Tavares

Claudio Manoel de Brito

Daniela Godoi Gonçalves

Debora Bevilaqua Grossi

Deusvenir de Souza Carvalho

Djacir Dantas Pereira de Macedo

Éder Cassio Rocha Ribeiro

Edson Amaro Junior

Elcio Juliato Piovesan

Elder Machado Sarmento

Eliana Meire Melhado

Eliova Zukerman

Erasmo Barros Da Silva

Fabíola Dach Éckeli

Fernando Kowacs

Frederico Mota Gonçalves Leite
\end{abstract}

Getulio Daré Rabello

Henrique Carneiro Campos

Hilton Mariano da Silva Junior

Jackeline Soráya Soares Barbosa

Jano Alves de Souza

Jayme Antunes Maciel Jr

João José Freitas de Carvalho

Jorge Von Zuben

José Geraldo Speciali

José Lucio de Oliveira Dantas

José Luiz Peixoto

José Stechman Neto

José Tadeu Tesseroli de Siqueira

Juliana Stuginski Barbosa

Karen dos Santos Ferreira

Liselotte Manke Barea

Luciana Campaner Fernandes

Luiz Paulo de Queiroz

Marcelo C. Ciciarelli

Marcelo Masruha Rodrigues

Marcelo Moraes Valença

Marco Antonio Arruda

Marco Antonio Minchola Robles

Maria Eduarda Nobre D. M. Costa

Mário F. P. Peres

Maurice Borges Vincent
Mauro Eduardo Jurno

Norma Regina Pereira Fleming

Orlando José Martins

Oswaldo Couto Jr.

Paulo Cesar R. Conti

Paulo Helio Monzillo

Pedro André Kowacs

Pedro Augusto Sampaio Rocha Filho

Pedro Ferreira Moreira Filho

Rafael Akira Murayama

Rafael dos Santos Silva

Raimundo Pereira da Silva Neto

Renan Barros Domingues

Renata Campi de Andrade Pizzo

Renata Silva Melo Fernandes

Reynaldo Leite Martins Jr.

Ricardo Tanus Vale

Sandro Blasi Esposito

Sidney Sredni

Silvia Regina Dowgan Tesseroli

de Siqueira

Stelio Leal Peçanha

Thaís Rodrigues Villa

Wagner de Oliveira

Wilson Luiz Sanvito 


\section{Congresso Brasileiro de Cefaleia Programa Científico}




\section{International Headache Society: The Headache-Masters School Scientific Program}

\section{RIO DE JANEIRO ROOM 13/09/2011}

08:20 - 12:10 Chair: Luiz Paulo de Queiroz - SC

TIME

08:00 - 08:20

$08: 20-08: 50$

$08: 50-09: 20$

$09: 20-09: 50$

09:50 - 10:20

$10: 20-10: 40$

$10: 40-11: 10$

$11: 10-11: 40$

$11: 40-12: 10$

$12: 10-13: 40$

$13: 40-18: 30$

$13: 40-14: 10$

$14: 10-14: 40$

$14: 40-15: 20$

$15: 20-15: 40$

09:50 - 10:20

$15: 40-16: 00$

$16: 00-16: 30$

$16: 30-17: 00$

$17: 00-17: 40$

$17: 40-18: 10$

$18: 10-18: 30$

\section{THEME - SPEAKER}

Subscriptions

History and Epidemiology of Headache, worldwide and in Brazil

- Luiz Paulo de Queiroz - SC

Pathophysiology of migraine - Robert Shapiro - USA

Clinical Imaging of Headache - Allan Purdy - USA

Migraine: Other Clinical Investigations - Jano Alves de Souza - RJ

Coffee-break

Migraine with aura and Migraine without aura - clinical aspects

- Luiz Paulo de Queiroz - SC

Other varieties of migraine - Marcelo C. Ciciarelli - SP

Discussions

Lunch

Chair: Jackeline Soráya Soares Barbosa - RJ

Headache and the child - Marco Antonio Arruda - SP

Migraine and the child - Marco Antonio Arruda - SP

TTH - Elder Machado Sarmento - RJ

Pathophysiology of TACs - Alan Rapoport - USA

Migraine: Other Clinical Investigations - Jano Alves de Souza - RJ

Coffee-break

Cluster headache clinical aspects and treatment - Alan Rapoport - USA

Other TACs - Michel Volcy - Colombia

Cough, exertional, sexual activity headaches and Thunderclap headache

- Benjamim Frishberg - USA

Primary stabbing headache, Hypnic headache and New daily-persistent headache (NDPH) - Claudio Manoel Brito - RJ

Discussions 


\title{
International Headache Society: The Headache-Masters School Scientific Program
}

\section{RIO DE JANEIRO ROOM \\ $14 / 09 / 2011$}

\author{
08:20 - 12:10 Chair: Elder Machado Sarmento - RJ
}

TIME

08:00 - 08:20

08:20 - 08:50

08:50 - 09:20

09:20 - 09:50

09:50 - 10:20

$10: 20-10: 40$

$10: 40-11: 10$

$11: 10-11: 40$

$11: 40-12: 10$

$12: 10-13: 40$

$13: 40-18: 30$

$13: 40-14: 10$

$14: 10-14: 40$

$14: 40-15: 10$

$15: 10-15: 40$

$15: 40-16: 00$

$16: 00-16: 30$

$16: 30-17: 00$

$17: 00-17: 40$

$17: 40-18: 10$

18:10 - 18:30

\section{THEME - SPEAKER}

Headache atribbuted to: head trauma, CNS infections (emphasis on the red flags) - Jano Alves de Souza - RJ

Biofeedback: what it is and its use in Headache Therapy - Luciana Campaner - SP

Cervicogenic Headache - José Geraldo Speciali - SP

Headache attributed to non-vascular intracranial disorder (emphasis on CSF pressure disorders) - Roberto Shapiro - USA

Headache attributed to disorder of homoeostasis - Benjamim Frishberg - USA

Coffee-break

Cranial neuralgias and central causes of facial pain - José Geraldo Speciali - SP

Co-morbidities of migraine - Robert Cowan - USA

Discussions

Lunch

\section{Chair: Fabíola Dach Éckeli - SP}

Anatomy of head pain - Morris Levin - USA

Taxonomy: How many headache disorders? Which of them are often seen?

- Morris Levin - USA

Chronic migraine and medication overuse headaches

- Hans-Chistoph Diener - Germany

Acute therapy - Alan Rapoport - USA

Coffee-break

Behavioral therapy - Steve Baskin - USA

Preventive therapy - Allan Purdy - USA

In hospital: Losing the fear of assessing a headache patient (usually secondary and with systemic diseases) - Fabíola Dach Eckeli - SP

ER: Losing the fear of assessing a headache patient (red flags, work out)

- Getúlio Daré Rabello - SP

Tertiary care unit: Losing the fear of assessing a headache patient

- Carlos Alberto Bordini - SP 


\section{1 st Brazilian - North American Headache Symposium (BRANAS) Part I: Advanced topics in Headache Medicine}

\section{SALAO NOBRE}

\section{$15 / 09 / 2011$}

07:00 - Entrega de materiais

Presidente honorário: Eliova Zukerman - SP

Secretário: Luiz Paulo de Queiroz - SP

\section{HORÁRIO}

08:10 - 08:20

08:20 - 08:40

08:40 - 09:10

09:10 - 09:30

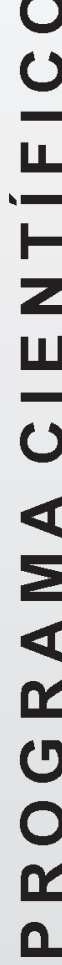

$09: 30-10: 00$

10:00 - 10:20

$10: 20-10: 40$

$10: 40-11: 10$

$11: 10-11: 30$

$11: 30-12: 00$

$12: 00-12: 20$

$12: 20-13: 40$

$12: 20-13: 40$

Cefaliatria atu
$14: 00-14: 40$

$14: 40-15: 00$

15:00 - 15:20

$15: 20-15: 40$

15:40 - 16:00

16:00 - 16:20

$16: 20-16: 40$

16:40 - 17:00

17:20 - 18:00

$18: 00-19: 20$
Abertura - Alan Rapoport - EUA; Carlos A. Bordini - SP; Eliova Zukerman - SP

Razões de disparidades na prevalência da migrânea nas diferentes regiões do país - Luiz Paulo de Queiroz - SC

The chronobiology of migraine - Robert Shapiro - EUA

Cefaleia no homem e cefaleia na mulher. Quais prevalecem em quem?

- Eliana Meire Melhado - SP

Migraine triggers, activators and natural history (with cases) - Robert Cowan - EUA Intervalo

Relação entre dor muscular e cefaleia - José Geraldo Speciali - SP

The eye and headache - Benjamim Frishberg - EUA

Modelos experimentais (animais e humanos) para pesquisa em cefaleias

- Marcelo Moraes Valença - PE

Classification and diagnosis of interesting headache cases - Morris Levin - EUA

Final Remarks - Eliova Zukerman - SP

Intervalo almoço

\section{Congresso Brasileiro de Cefaleia}

\section{Abertura}

Presidente: Luiz Paulo de Queiroz - SC

Migraine and stroke - Hans-Christoph Diener - ALE

Síndromes das dores disfuncionais - Jano Alves de Souza - RJ

Imagens em cefaleia - Edson Amaro Jr. - SP

Peptídeos e cefaleia - Marcelo C. Ciciarelli - SP

Triptanas: ainda o padrão ouro na crise migranosa - Mauro Eduardo Jurno - MG

Intervalo

Quebrando paradigmas no tratamento da migrânea - Carlos Alberto Bordini - SP

Cefaliatria: armadilhas em que não caio - Pedro Ferreira Moreira Filho - RJ

Ten Things Everyone Should Know about Headache Medicine - Allan Purdy - EUA

Visita oficial à sessão de pôsteres - área expositiva Sala Goiás

Coquetel 


\title{
XXV Congresso Brasileiro de Cefaleia
}

\section{SALÃO NOBRE}

\section{$16 / 09 / 2011$}

\author{
Conferência \\ Presidente: Mário F. P. Peres - SP
}

\section{HORÁRIO TEMA - PALESTRANTE}

08:00 - 09:00 Tratamento profilático da Migrânea Crônica com BOTOX® (OnabotulinumtoxinA) - Stephen D. Silberstein - EUA

09:00 - 09:40 Migraine Pipeline in 2011 - Alan Rapoport - EUA

Sessão de discussão de casos: Outras cefaleias primárias

Presidente: Célia Aparecida de Paula Roesler - SP

09:40 - 10:00 Cefaleia primária da tosse e cefaleia primária do esforço e da atividade sexual

- Ariovaldo Alberto da Silva Jr - MG

10:00 - 10:20 Intervalo

10:20 - 10:40 Cefaleia hípnica - Ana Luiza Lima Antoniazzi - SP

10:40 - 11:00

Cefaleia em trovoada primária - Cláudio Manoel de Brito - RJ

$11: 00-11: 20$

Cefaleia nova e persistente diária - Ana Maria Ladeira Yamada - RJ

$11: 20-11: 40$

Hemicrânia contínua - Henrique Carneiro de Campos - MG

\section{Conferência}

Presidente: Orlando José Martins - SP

11:40 - 12:20 Difficult headaches - Hans-Christoph Diener - ALE

12:20 - 13:35 Simpósio Allergan

Sessão de discussão de casos: cefaleias trigêmino-autonômicas

Presidente: Erasmo Barros da Silva - PB

13:40 - 14:00 Cefaleia em salvas - Alexandre Ottoni Kaup - SP

Cefaliatria atual I

Presidente: José Lucio de Oliveira Dantas - SE

$14: 00-14: 20$

Cefaleia em salvas - Elder Machado Sarmento - RJ

$14: 20-14: 40$

Hemicrânias paroxísticas \& SUNCT - Maria Eduarda Nobre M. D. Costa - RJ 


\section{Congresso Brasileiro de Cefaleia}

\section{SALAO NOBRE 16/09/2011}

Sessão de discussão de casos: CTT e migrânea Presidente: Osvaldo Couto Jr - SP

\section{HORÁRIO TEMA - PALESTRANTE}

14:40 -15:20 Chronic migraine (case discussions) - Alan Rapoport - EUA Allan Purdy - EUA

15:20 - 15:40 Migrânea episódica - Raimundo Pereira da Silva Neto - PI

15:40 - 16:00 CTT crônica - Marco Antonio Minchola Robles - PR

$16: 00-16: 20$ Intervalo

16:20 - 16:40 CTT episódica - Cláudia Tavares de Souza - MG

Conferência

Presidente: Sidney Sredni - SP

16:40 - 17:00 Migrânea crônica e o espectro bipolar - Mário F. P. Peres - SP

17:00 - 17:20 Valor preditivo de Tomografia computadorizada e Ressonância Nuclear Magnética

- Paulo Hélio Monzillo - SP

17:20 - 18:00 Os paradigmas da medicina - Wilson Luiz Sanvito - SP

18:00 - 19:00 Assembléia da SBCe

\section{SALAO NOBRE}

\section{Cefaléia nas crianças}

Presidente: Célia Aparecida de Paula Roesler - SP

\section{HORÁRIO TEMA - PALESTRANTE}

08:00 - 08:20

$08: 20-08: 40$

08:40 - 09:00

09:00 - 09:20

09:20 - 09:40

09:40 - 10:00

$10: 00-10: 20$
Abordagem da cefaléia na emergência - Marcelo Masruha Rodrigues - ES

Epidemiologia da cefaléia infância - Marco Antonio Arruda - SP

Sono em crianças migranosas - Thaís Rodrigues Villa - SP

Qualidade de vida em cefaléia infância - Liselotte Manke Barea - RS

Psicopatologia e cefaléia infância - Deusvenir de Souza Carvalho - SP

Terapêutica da migrânea na infância - Sandro Blasi Spósito - SP

Intervalo 


\title{
XXV Congresso Brasileiro de Cefaleia
}

\section{SALÃO NOBRE}

$17 / 09 / 2011$

\author{
Cefaliatria atual II \\ Presidente: Stélio Leal Peçanha - SP
}

\section{HORÁRIO I TEMA - PALESTRANTE}

10:20 - 10:40 Arterites da artéria temporal menos comuns (não as de células gigantes)

- Renan Barros Domingues - ES

10:40 - 11:00 Qualidade de vida em migranosos e epilépticos - Hilton Mariano da Silva Jr - SP

11:00-11:20 Genética e cefaleia - Elcio Juliato Piovesan - PR

11:20 - 11:40 Cefaleia hipertensão intracraniana idiopática - Éder Cássio Rocha Ribeiro - GO

11:40-12:00 Cefaleia e vestibulopatias - Fabíola Dach Éckeli - SP

12:00 - 12:20 Cefaleia e endocrinopatias - Pedro Augusto Sampaio Rocha Filho - PE

12:20 - 13:40 Intervalo - Almoço

$13: 40-15: 00$

Apresentações orais - Coordenação do grupo de prêmios - José Geraldo Speciali - SP

Cefaliatria atual III

Presidente: Cláudia Tavares de Souza - MG

15:00 - 15:20 Opioides em cefaleias primárias: nem tanto ao céu nem tanto ao inferno

- Diacir Dantas P. Macedo - RN

$15: 20-15: 40$

Estimulação magnética transcutânea e dor - Pedro Kovacs - PR

$15: 40-16: 00$

Cefaleia na sala de emergência - João José Freitas de Carvalho - CE

$16: 00-16: 20$

Intervalo

O melhor tema do ano passado

Presidente: Célia Aparecida de Paula Roesler - SP

$16: 20-16: 40$

Sempre quis falar sobre... A cefaleia da hipotensão liquórica espontânea é rara?

- Getúlio Daré Rabello - SP

$16: 40-17: 00$

Sempre quis falar sobre... A neuromodulação periférica no tratamento das cefaleias refratárias: o que aconteceu até agora e o que esperar do futuro - Fernando Kowacs - RS

$17: 00-17: 20$ Sempre quis falar sobre... Cefaleias atípicas - Jayme Antunes Maciel Jr - SP

$17: 20-17: 40$ Sempre quis falar sobre... O inefável orbe do mururu - Maurice Borges Vincent - RJ

$17: 40-18: 00$ Enxaqueca é dor neuropática ou nociceptiva? - Norma Regina Pereira Fleming - RJ

18:00 -18:10 Encerramento

$22: 30$

Festa de confraternização 


\section{Congresso do Comitê de Dor Orofacial da SBCe}

\section{SALA RIO DE JANEIRO}

08:20 - 10:40 - Coordenadora: Renata Campi de Andrade Pizzo - SP

\section{HORARIO}

08:20 - 09:05

09:05 - 09:45

09:45 - 10:15

10:15 - 10:20

$10: 20-10: 40$

\section{TEMA - PALESTRANTE}

O que há sobre DTM baseado em evidência científica e a prática clínica

- Paulo Cesar R. Conti - SP

Genética e dor orofacial: perspectivas futuras - Paulo Cesar R. Conti - SP

Mecanismos da dor orofacial - Norma Regina Pereira Fleming - RJ

Discussão

Intervalo

10:40 - 12:20 - Coordenadora: Juliana Stuginski Barbosa - SP

$(10: 40-11: 10$

Estado da arte sobre a relação fibromialgia e DTM - Rafael Akira Murayama - SP

$11: 10-11: 40$

Fatores de risco para a dor musculoesquelética mastigatória - Rafael dos Santos Silva - SP

$11: 40-12: 10$ Patologias reumatológicas sua relação com a DTM - Renata Silva M. Fernandes - PE

$12: 10-12: 20$ Discussão

$12: 20-13: 40$

Intervalo - Almoço

\section{SALÃO NOBRE}

$13: 40-14: 00$

Abertura

\section{SALA RIO DE JANEIRO}

14:00 - 17:30 - Coordenador: Frederico Gonçalves Mota Leite - MG

$14: 00-14: 30$

Tratamento Fibromialgia e DTM: o que existe em comum? - Amélia Pasqual Marques - SP

$14: 30-15: 00$

Evidência do tratamento fisioterapêutico na cefaleia e DTM - Debora Bevilaqua Grossi - SP

$15: 00-15: 30$ Importância clínica das placas oclusais no tratamento das DTM - Ricardo Tanus Vale - MG

$15: 30-15: 40$ Discussão

$15: 40-16: 00$ Intervalo 


\section{Congresso do Comitê de Dor Orofacial da SBCe}

\section{SALA RIO DE JANEIRO 15/09/2011}

16:00 - 17:30 - Coordenadora: Renata Silva Melo Fernandes - PE

\section{HORARIO | TEMA - PALESTRANTE}

16:00 - 16:30 A importância da estabilização ortopédica da oclusão nas DTMs - Wagner de Oliveira - SP

16:30 - 17:30 Manejo clínico dos vários estágios do disco articular - Ricardo Tanus Vale - MG

17:00 - 17:30 Diagnóstico por imagem: tomografia computadorizada e ressonância magnética

- Reynaldo Martins - MT

18:00 - 19:20 Visita - Discussão de pôsteres na Área Expositiva

Coquetel

\section{SALA RIO DE JANEIRO}

\section{$16 / 09 / 2011$}

08:20 - 09:50 Coordenador: Ricardo Tanus Vale - MG

$08: 20-08: 50$

SAB e odontalgia atípica: como proceder o tratamento? - Jorge Von Zuben - SP

08:50 - 09:20

Articulação temporomandibular em pacientes geriátricos - José Stechman Neto - PR

$09: 20-09: 50$

Métodos intervencionistas em dor orofacial - Frederico G. Mota Leite - MG

09:50 - 10:00

Discussão

$10: 00-10: 20$

Intervalo

10:20 - 12:20 Coordenadora: Daniela Godoi Gonçalves - SP

10:20 - 10:50 O significado clínico do dignóstico diferencial em dores neuropáticas orofaciais

- José Tadeu Tesseroli de Siqueira - SP

10:50 - 11:20 Genética e Dor Crônica - José Geraldo Speciali - SP

11:20-11:50 Avaliação sensitiva nas dores orofaciais neuropáticas

- Silvia Regina Dowgan Tesseroli de Siqueira - SP

11:50-12:20 Neuralgias de ramos terminais do trigêmeo: o que o dentista deve saber

- Renata Campi de Andrade Pizzo - SP

12:20 - 13:20 Intervalo - Almoço 


\section{Congresso do Comitê de Dor Orofacial da SBCe}

\section{SALA RIO DE JANEIRO 16/09/2011}

13:30 - 16:00 Coordenador: José Stechman Neto - PR

HORÁRIO | TEMA - PALESTRANTE

13:30 - 14:00 Diagnóstico dos Distúrbios do sono no paciente com dor orofacial: como proceder na clínica - Cibele Dal Fabbro - SP

14:00 - 14:30 Há relação entre bruxismo do sono, DTM e cefaleias primárias?

- Cinara Maria Camparis - SP

14:30 - 15:00 Bruxismo do sono: opções atuais de tratamento - Juliana Stuginski Barbosa - SP

$15: 00-15: 30$

$15: 30-15: 40$

$15: 40-16: 00$

DTM x SAOS: o uso do aparelho intraoral - Ana Cristina Lotaif - SP

Discussão

16:00 - 18:10 Coordenador: Jorge Von Zuben - SP

16:20 - 16:30 Contribuição das DTMs na cronificação das cefaleias primárias

- Daniela Godoi Gonçalves - SP

16:30 - 17:00 Cefaleia secundária a DTM de origem muscular ou CTT? Qual o diagnóstico?

- José Luiz Peixoto - SP

17:00 - 17:30 Diferença entre homem e mulher em relação à percepção da dor

- Karem dos Santos Ferreira - SP

17:30 - 18:00 Mesa-redonda: Pergunte aos especialistas presentes

\section{SALÃO NOBRE}

\section{3:40 - 15:00 Apresentações orais}




\section{Congresso Brasileiro de Cefaleia Temas Livres - Apresentação Oral}




\title{
CE 06 - Alodínia cutânea em pacientes com migrânea crônica: frequência e severidade
}

\author{
Gonçalves MC, Florencio LL, Chaves TC, Dach F, Speciali JG, Bevilaqua Grossi D
}

Faculdade de Medicina de Ribeirão Preto - Universidade de São Paulo

Introdução: A cefaleia é um dos sintomas mais comuns visto na população, cerca de $76 \%$ das mulheres e $57 \%$ dos homens tem pelo menos um ataque por mês. ' Entre as formas mais recorrentes de cefaleia está a migranea, que pode começar como uma doença episódica, ocorrendo menos de 15 dias por mês, mas também pode evoluir para uma forma crônica e ocorrer por 15 dias ou mais. Pacientes com migrânea apresentam alta prevalência de alodínia cutânea $(\mathrm{AC}){ }^{8}$ sintoma definido como dor em reposta a um estímulo não nociceptivo aplicado sobre a pele normal. Recentemente a relação entre migrânea e $A C$ vem atraindo maior interesse, uma vez que esta foi reconhecida como um sinal de sensibilização central durante as crises migranosas ${ }^{3}$ e sugerida como um fator de risco para a cronificação da migrânea. ${ }^{2}$ Além disso, tem sido sugerido que a $A C$ pode influenciar na resposta ao tratamento com triptanos, ${ }^{4}$ provocando influência negativa na eficácia do tratamento, ${ }^{4}$ interferindo na medicação que deve ser administrada ${ }^{5}$ e ainda há relato de que os triptanos podem induzir o surgimento da AC 6 . Objetivo: Avaliar a presença e a severidade da alodínia cutânea em portadores de migrânea crônica. Metodologia: Foi avaliada a frequência e a severidade da AC, através da versão brasileira ${ }^{7}$ do questionário 12 item Allodynia Symptom Cheklist (ASC-12) (Figura 1) em 18 voluntários (16 mulheres), alfabetizados, com idade média $40 \pm 12$, portadores de migrânea crônica diagnosticados de acordo com a Classificação Internacional de Cefaleias por neurologistas especialistas. O ASC-12 é um questionário autoaplicável cuja pontuação varia de 0-24 pontos e classifica o indivíduo como: sem alodínia (0-2 pontos), leve (3-5 pontos), moderada (6-8 pontos) e severa (9 ou mais pontos). Foram excluídos voluntários com diagnóstico concomitante de outras cefaléias, fibromialgia, neuralgia do trigêmio e doenças sistêmicas que apresentem neuropatia periférica sensitiva. Este trabalho foi aprovado pelo Comitê de Ética em Pesquisa da Faculdade de Medicina de Ribeirão Preto-USP processo $n^{\circ} 13470 / 2010$ e todos os voluntários assinaram o termo de consentimento livre e esclarecido. Resultados: Um total de dezesseis (89\%) dos dezoito indivíduos avaliados apresentaram alodinia cutânea, sendo a maioria classificada como severa (61\%).

\begin{tabular}{|c|c|c|c|c|c|}
\hline $\begin{array}{l}\text { 1. Durante sua dor de cabeça mais forte, vocé sente incómodo ao pentear ou } \\
\text { escovar seu cabelo? }\end{array}$ & $\begin{array}{l}\text { ( ) } \\
\text { Essa situação não } \\
\text { se aplica a mim }\end{array}$ & $\begin{array}{r}\text { Não, } \\
\text { nunca }\end{array}$ & $\underset{\text { Raramente }}{(\text { ) }}$ & $\begin{array}{l}(\stackrel{)}{()} \\
\text { Ás vezes sim, } \\
\text { Ás vezes não }\end{array}$ & $\begin{array}{c}\left(\begin{array}{c}) \\
\text { A maior parte } \\
\text { das vezes }\end{array}\right. \\
\end{array}$ \\
\hline $\begin{array}{l}\text { 2. Durante sua dor de cabeça mais forte, vocé sente incômodo ao prender seu } \\
\text { cabelo (por exemplo, rabo de cavalo)? }\end{array}$ & $\begin{array}{l}\text { ( ) } \\
\text { Essa situação não } \\
\text { se aplica a mim }\end{array}$ & $\begin{array}{r}\text { ( Não, } \\
\text { nunca }\end{array}$ & $\begin{array}{l}(\text { ) } \\
\text { Raramente }\end{array}$ & 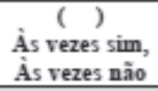 & $\begin{array}{c}(\text { ) } \\
\text { A maior parte } \\
\text { das vezes }\end{array}$ \\
\hline 3. Durante sua dor de cabeça mais forte, você sente incômodo ao fazer a barba? & $\begin{array}{c}\text { ( ) } \\
\text { Essa situação não } \\
\text { se aplica a mim }\end{array}$ & $\begin{array}{r}\text { ( Não, } \\
\text { nunca }\end{array}$ & 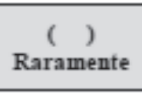 & $\begin{array}{l}(\quad) \\
\text { As vezes sim, } \\
\text { Ás vezes não }\end{array}$ & 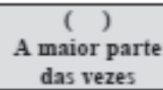 \\
\hline 4. Durante sua dor de cabeça mais forte, você sente incômodo ao usar óculos? & $\begin{array}{l}\text { ( ) } \\
\text { Essa situação não } \\
\text { se aplica a mim }\end{array}$ & $\begin{array}{r}\text { ( Não, } \\
\text { nunca }\end{array}$ & $\stackrel{(\text { ( ) }}{\text { Raramente }}$ & $\begin{array}{l}(\quad) \\
\text { Às vezes sim, } \\
\text { Ás vezes não }\end{array}$ & 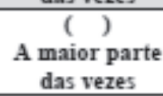 \\
\hline $\begin{array}{l}\text { 5. Durante sua dor de cabeça mais forte, você sente incômodo ao usar lentes de } \\
\text { contato? }\end{array}$ & $\begin{array}{l}\text { ( ) } \\
\text { Essa situação não } \\
\text { se aplica a mim }\end{array}$ & $\begin{array}{r}\text { ( Nào, } \\
\text { nunca }\end{array}$ & $\stackrel{(\text { ( ) }}{\text { Raramente }}$ & 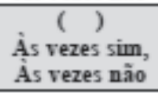 & $\begin{array}{c}\left(\begin{array}{c}) \\
\text { A maior parte } \\
\text { das vezes }\end{array}\right. \\
\end{array}$ \\
\hline 6. Durante sua dor de cabeça mais forte, você sente incômodo ao usar brincos? & $\begin{array}{l}\text { ( ) } \\
\text { Essa situação não } \\
\text { se aplica a mim }\end{array}$ & $\begin{array}{r}\text { ( Não, } \\
\text { nunca }\end{array}$ & 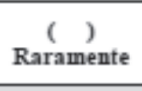 & $\begin{array}{l}(\underset{)}{(}) \\
\text { Às vezes sim, } \\
\text { Ás vezes não }\end{array}$ & $\begin{array}{c}(\text { ) } \\
\text { A maior parte } \\
\text { das vezes }\end{array}$ \\
\hline 7. Durante sua dor de cabeça mais forte, você sente incômodo ao usar colar? & $\begin{array}{l}\text { ( ) } \\
\text { Essa situação não } \\
\text { se aplica a mim }\end{array}$ & $\begin{array}{r}\text { ( Não, } \\
\text { nunca }\end{array}$ & $\begin{array}{l}\text { ( ) } \\
\text { Raramente }\end{array}$ & $\begin{array}{l}\left(\begin{array}{l}() \\
\text { Às vezes sim, } \\
\text { Ás vezes não }\end{array}\right.\end{array}$ & $\begin{array}{c}\left(\begin{array}{l}) \\
\text { A maior parte } \\
\text { das vezes }\end{array}\right. \\
\text {. }\end{array}$ \\
\hline $\begin{array}{l}\text { 8. Durante sua dor de cabeça mais forte, você sente incômodo ao usar roụas } \\
\text { justas? }\end{array}$ & $\begin{array}{c}\text { ( ) } \\
\text { Essa situação não } \\
\text { se aplica a mim }\end{array}$ & $\begin{array}{r}\text { ( Não, } \\
\text { nunca }\end{array}$ & $\stackrel{(\text { ( ) }}{\text { Raramente }}$ & 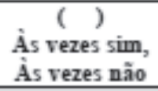 & $\begin{array}{c}\left(\begin{array}{l}) \\
\text { A maior parte } \\
\text { das vezes }\end{array}\right. \\
\end{array}$ \\
\hline $\begin{array}{l}\text { 9. Durante sua dor de cabeça mais forte, você sente incômnodo ao tomar banho } \\
\text { (por exemplo, quando a água do chuveiro cai no seu rosto)? }\end{array}$ & $\begin{array}{c}\text { ( ) } \\
\text { Essa situação não } \\
\text { se aplica a mim }\end{array}$ & $\begin{array}{r}\left(\begin{array}{l}\text { ( ) } \\
\text { Nào, } \\
\text { nunca }\end{array}\right. \\
\end{array}$ & $\stackrel{(\text { ( ) }}{\text { Raramente }}$ & $\begin{array}{l}(\quad) \\
\dot{A} \text { s vezes sim, } \\
\text { Ás vezes não }\end{array}$ & $\begin{array}{c}\left(\begin{array}{l}) \\
\text { A maior parte } \\
\text { das vezes }\end{array}\right.\end{array}$ \\
\hline $\begin{array}{l}\text { 10. Durante sua dor de cabeça mais forte, você sente incômodo ao apoiar seu } \\
\text { rosto ou cabeça em um travesseiro ou almofada? }\end{array}$ & $\begin{array}{c}\text { ( ) } \\
\text { Essa situação não } \\
\text { se aplica a mim }\end{array}$ & $\begin{array}{r}\text { ( Não, } \\
\text { nunca }\end{array}$ & 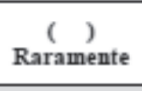 & $\begin{array}{l}\left(\begin{array}{l}() \\
\text { Ass vezes sim, } \\
\text { As vezes não }\end{array}\right. \\
\end{array}$ & $\begin{array}{c}\left(\begin{array}{c}) \\
\text { A maior parte } \\
\text { das vezes }\end{array}\right. \\
\end{array}$ \\
\hline $\begin{array}{l}\text { 11. Durante sua dor de cabeça mais forte, você sente incômodo ao se expor ao } \\
\text { calor (por exemplo, cozinhar, lavar seu rosto com água quente)? }\end{array}$ & $\begin{array}{c}\text { ( ) } \\
\text { Essa situação não } \\
\text { se aplica a mim }\end{array}$ & $\begin{array}{r}\text { ( Não, } \\
\text { nunca }\end{array}$ & $\stackrel{(\text { ( ) }}{\text { Raramente }}$ & 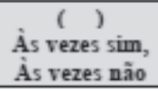 & $\begin{array}{c}\left(\begin{array}{l}) \\
\text { A maior parte } \\
\text { das vezes }\end{array}\right. \\
\end{array}$ \\
\hline $\begin{array}{l}\text { 12. Durante sua dor de cabeça mais forte, você seute incômodo ao se expor ao } \\
\text { frio (por exemplo, usar uma bolsa de gelo, lavar seu rosto com água fria)? }\end{array}$ & $\begin{array}{l}\text { ( ) } \\
\text { Essa situação não } \\
\text { se aplica a mim }\end{array}$ & $\begin{array}{r}\text { ( }) \\
\text { Não, } \\
\text { nunca }\end{array}$ & $\begin{array}{c}(\quad) \\
\text { Raramente }\end{array}$ & $\begin{array}{l}(\text { ( ) } \\
\text { Às vezes sim, } \\
\text { Ás vezes não }\end{array}$ & $\begin{array}{c}(\text { ) } \\
\text { A maior parte } \\
\text { das vezes }\end{array}$ \\
\hline Pontuação total & & & & & \\
\hline
\end{tabular}




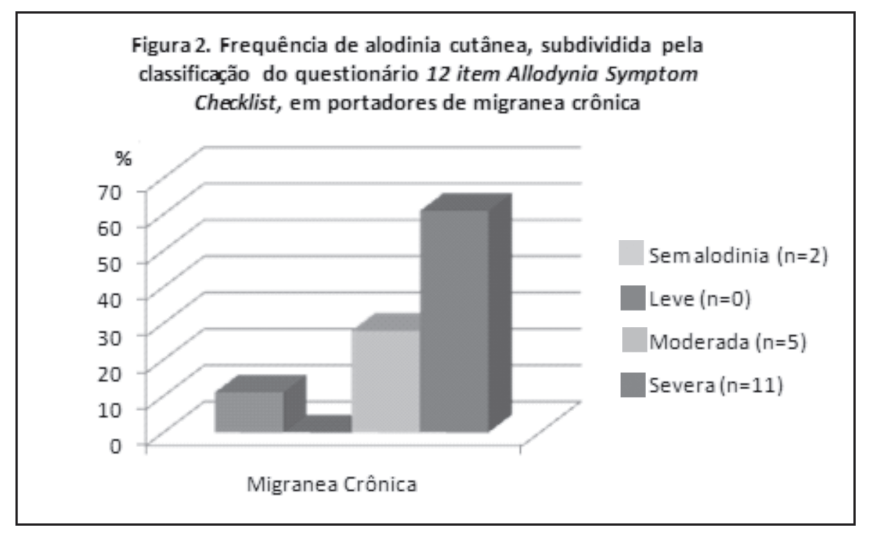

Não houve correlação, verificada através da Correlação de Spearman, entre a pontuação do questionário e a idade (- 0,095), o tempo de doença $(21 \pm 14$ anos) $(-0,12)$ e o Índice de Massa Corporal $(25 \pm 5)(-0,11)$. Discussão: Os indivíduos com migrânea crônica, na amostra estudada, apresentaram alta frequência e severidade de $A C$, demonstrando que esse sintoma persiste na fase crônica podendo estar envolvido na cronificação dessa doença. ${ }^{2} \mathrm{~A}$ maioria dos indivíduos foi classificada com AC severa, indicando que os pacientes não apenas tem o sintoma, mas que este também é severo sugerindo a importância de sua avaliação na prevenção da cronificação da migranea. Conclusão: $A$ frequência da $A C$ em portadores de migrânea crônica é elevada, sendo a forma severa a mais prevalente, sugerindo a importância da avaliação desse sintoma em migranosos crônicos.

Referências:

1. Lipton, R.B.; Bigal, M.E. Tem Lessons on epidemiology of migraine. Headache, v.47, p.S2-S9,2007.

2. Guy N, Marques AR, Orliaguet T, Lanteri-Minet M, Dallel R, Clavelou P. Are there differences between cephalic and extracephalic cutaneous allodynia in migraine patientes?. Cephalalgia $2010 \mathrm{Jul} ; 30(7): 881-6$.

3. Dodick D, Silberstein S. Central sensitization theory of migraine: clinical implications. Headache 2006; 46(4):S182-S191.

4. Burstein R, Jakubowski M. Analgesic triptan action in an animal model of intracranial pain: a race against the development of central sensitization. Annals of Neurology 2004; 55: 27-36.

5. Lampl C, Huber G, Haas S, Rittberger E, Diener H. Difference in triptan effect in patients with migraine and early allodynia. Cephalalgia 2008 Oct;28(10): $1031-8$.

6. Linde M, Elam M, Lundblad L, Olausson H, Dahlöf CG. Sumatriptan (5-HT1B/1Dagonist) causes a transient allodynia. Cephalalgia 2004;24:1057-66.

7. Florencio LL, Gonçalves MC, Chaves TC, Dach FE, Speciali JG, Bevilaqua-Grossi D. Adaptação transcultural do 12 item Allodynia Symptom Cheklist - versão brasileira. Projeto de mestrado com bolsa FAPESP (2010/03377-0) e auxílio pesquisa CNPq (2009/17580-4).

8. Ashkenazi A, Silberstein S, Jakubowski M, Burstein R. Improved Identification of Allodynic Migraine Patients Using a Questionnaire. Cephalalgia 2007 27: 325-9.

\title{
CE 13 - Validação da osmofobia como critério diagnóstico de migrânea
}

\author{
Silva Neto RP, Almeida KJ, Valença MM \\ Centro de Neurologia e Cefaleia do Piauí - Teresina, PI, Brasil
}

Introdução: A osmofobia é definida como uma intolerância aos odores e é um sintoma associado apenas às cefaleias primárias, principalmente à migrânea com ou sem aura. ${ }^{1,2}$ Porta-Etessam et al. $(2009)^{3}$ referiram osmofobia em $54,0 \%$ dos pacientes com migrânea e em nenhum paciente com cefaleia do tipo tensional (CTT). No estudo de Zanchin et al. (2005)², a osmofobia esteve presente em 43,0\% dos pacientes com migrânea sem aura, 39,0\% na migrânea com aura, e em 7,0\% na cefaleia em salvas, mas não foi observada em qualquer paciente com CTT ou outra cefaleia primária. Corletto et al. (2008) ${ }^{4}$ encontraram uma prevalência de osmofobia de $25,1 \%$ durante as crises de cefaleia em pacientes com migrânea e 8,3\%, nos paciente com CTT. Esse estudo demonstrou que a osmofobia é um sintoma de baixa sensibilidade e de alta especificidade, mais do que fotofobia ou fonofobia, para o diagnóstico diferencial entre migrânea e CTT. Objetivos: Avaliar a associação entre osmofobia e diagnóstico de migrânea e determinar a sensibilidade, a especificidade e os valores preditivo positivo e negativo da osmofobia como critério diagnóstico de migrânea. Método: Estudo prospectivo e transversal realizado em uma clínica especializada em cefaleia, para avaliar a osmofobia associada à cefaleia numa amostra, aleatória e por conveniência, de 50 pacientes com migrânea e 50 com cefaleia do tipo tensional (controle), de acordo com a Sociedade Internacional de Cefaleia ${ }^{5}$, obedecendo a critérios de inclusão e exclusão. A análise estatística foi realizada por teste do qui-quadrado, com correção de Yates, e pelo teste † de Student para comparação de médias, admitindo-se nível de significância de $p<0,05$. Foram determinados os parâmetros de sensibilidade, especificidade, valor preditivo positivo, valor preditivo negativo, concordância (índice kappa), acurácia e odds ratio. Este estudo foi aprovado pelo Comitê de Ética em Pesquisas da Universidade Estadual do Piauí e todos os pacientes assinaram o termo de consentimento livre e esclarecido. Resultados: Foram estudados 50 pacientes com migrânea (46 mulheres e 4 homens, com média de idade de 37,10 19,05 anos) e 50 com CTT (19 mulheres e 31 homens, com média de idade de $40,06 \pm 10,45$ anos). As médias de idade não apresentaram diferença, estatisticamente, significante, refletindo a homogeneidade dos grupos em relação a esta variável. Dos 50 pacientes com migrânea, 86,0\% (43/50) apresentaram osmofobia, em comparação com 6,0\% (3/50) do controle (Tabelas 1 e 2).

Tabela 1. Dados amostrais de 50 pacientes com migrânea e 50 com cefaleia do tipo tensional

\begin{tabular}{lllll}
\hline & Migrânea & CTT & $\begin{array}{l}\text { Testes } \\
\text { estatísticos }\end{array}$ & $p$ \\
\hline $\mathrm{n}$ & 50 & 50 & & \\
\hline Feminino/masculino & $46 / 4$ & $19 / 31$ & $\chi^{2}=32,044$ & $<0,001$ \\
Média de idade (anos) & 37,10 & 40,06 & $\dagger=-1,514$ & 0,133 \\
Desvio padrão & 9,05 & 10,45 & & \\
\hline
\end{tabular}


Tabela 2. Sensibilidade e especificidade da osmofobia no diagnóstico de migrânea

\begin{tabular}{lll}
\hline & Migrânea & CTT \\
\hline Osmofobia $(+)$ & 43 & 3 \\
Osmofobia $(-)$ & 7 & 47 \\
\hline
\end{tabular}

A associação entre osmofobia e diagnóstico de migrânea foi, estatisticamente, significante $\left(\chi^{2}=61,23 ; p<0.0001\right)$, com uma concordância substancial $(k=0,80)$, uma acurácia precisa $(90,0 \%)$ e $\mathrm{OR}=96,2$ (Tabela 3).

Tabela 3. Associação entre osmofobia e o diagnóstico de migrânea.

\begin{tabular}{lll}
\hline Parâmetros & $\%$ & IC $95 \%$ \\
\hline Sensibilidade & 86,0 & $85,3-86,7$ \\
Especificidade & 94,0 & $93,5-94,5$ \\
Valor preditivo positivo & 93,5 & $93,0-94,0$ \\
Valor preditivo negativo & 87,0 & $86,3-87,7$ \\
\hline
\end{tabular}

Discussão: De acordo com os critérios diagnósticos da International Headache Society (2004), ${ }^{5}$ apenas náuseas, vômitos, fotofobia e fonofobia são sintomas associados à cefaleia durante a crise de migrânea. No entanto, a osmofobia, outra modalidade de hiperacuidade sensorial, é frequentemente relatada por pacientes migranosos, apesar de o seu exato mecanismo ser ainda desconhecido. Recentemente, um estudo com 60 pacientes com migrânea mostrou a prevalência dos principais sintomas associados durante a crise, descritos a seguir: náuseas (80,0\%), vômitos $(53,0 \%)$, fonofobia $(93,0 \%)$, fotofobia $(87,0 \%)$ e osmofobia $(81,7 \%), 6$ enquanto outros estudos evidenciaram prevalências menores de osmofobia (de 25,0 a 54,0\%). ${ }^{1,3,7} \mathrm{Em}$ nosso estudo, a osmofobia esteve presente em $86,0 \%$ dos pacientes com migrânea, concordando os achados de Siöstrand et al. $(2010)^{6}$ que encontraram $81,7 \%$. Sugere-se que, se a osmofobia ocorrer de forma consistente na migrânea, durante os ataques, então ela poderia fazer parte dos critérios diagnósticos para migrânea. Encontramos, também, 6,0\% de osmofobia nos pacientes com CTT, mas Zanchin et al. (2007), ${ }^{7}$ ao estudarem 198 pacientes com CTT, não encontraram osmofobia em nenhum paciente. Ainda observamos que a especificidade da osmofobia para o diagnóstico de migrânea é maior que a sensibilidade, que também é alta. Logo a ausência de osmofobia tende a afastar o diagnóstico de migrânea. Em dois estudos, a osmofobia mostrou uma baixa sensibilidade, mas uma alta especificidade no diagnóstico diferencial entre migrânea e CTT, mais do que fotofobia ou fonofobia. ${ }^{1,4}$ Conclusões: Houve correlação positiva entre presença de osmofobia e diagnóstico de migrânea. A acurácia da osmofobia para o diagnóstico de migrânea foi expressiva. A osmofobia como sintoma isolado tem alta sensibilidade e especificidade para o diagnóstico de migrânea e parece ser um marcador específico para diferenciá-la da CTT.

\section{Referências:}

1. Kelman L. Osmophobia and taste abnormality in migraineurs: a tertiary care study. Headache 2004;44(10): 1019-23.

2. Zanchin G, Dainese F, Mainardi F, Mampreso E, Perin C, Maggioni F. Osmophobia in primary headaches. J Headache Pain 2005;6(4):213-5.

3. Porta-Etessan J, Casanova I, García-Cobos R, Lapeña T, Fernández MJ, GarcíaRamos R, et al. Análisis de la osmofobia en las cefaleas primarias. Neurología 2009;24(5):315-7.

4. Corletto E, Dal Zotto L, Resos A, Tripoli E, Zanchin G, Bulfoni C. et al. Osmophobia in juvenile primary headaches. Cephalalgia 2008;28(8):825-31.

5. International Headache Society. Classification and diagnostic criteria for headache disorders, cranial neuralgias, and facial pain. Cephalalgia 2004;24(Suppl 1):1-160.

6. Siöstrand C, Savic I, Laudon-Meyer E, Hillert L, Lodin K, Walkdenlind E. Migraine and olfactory stimuli. Curr Pain Headache Rep 2010;14:244-51.

7. Zanchin G, Dainese F, Trucco M, Mainardi F, Mampreso E, Maggioni F. Osmophobia in migraine and tension-type headache and its clinical features in patients with migraine. Cephalalgia 2007;27(9):1

\title{
CE 20 - Frequent headaches in Brazilian school-aged children - a Nationwide Study
}

\author{
Arruda $M A^{\prime}$; Arruda R; Bigal ME $E^{3,4}$ \\ ${ }^{1}$ Director, Glia Institute, Ribeirao Preto, SP, Brazil; ${ }^{2}$ Graduate student, University of Campinas Medical School \\ ${ }^{3}$ Global Director for Scientific Affairs - Neuroscience, Merck Research Laboratories, Whitehouse Station, NJ, U.S. \\ ${ }^{4}$ Department of Neurology, Albert Einstein College of Medicine, Bronx, NY, U.S.
}

Introduction: The prevalence of frequent headaches in the preadolescent pediatric population is largely unknown. Adolescent studies found that $\mathrm{CDH}$ is a significant problem in this age group, and that substantial differences between adolescents and adults with $\mathrm{CDH}$ exist. ${ }^{2}$ Indeed, an increased biological risk in adolescents with $\mathrm{CDH}$ was suggested. ${ }^{2}$ For other neurological disorders, early onset cases have the highest level of biological or genetic risk. Very few studies investigated $\mathrm{CDH}$ s in the pre-adolescent population., 3-5 A recent population study conducted by us with a community sample showed that a sizable $1.7 \%$ of the children had chronic daily headaches and $4.2 \%$ of the children had 10 or more days of headache by month. ${ }^{6}$ To the best of our knowledge, studies assessing frequent episodic headache in a nationwide representative populational sample have not been published. Accordingly, herein we estimate the prevalence of chronic daily headache $(\mathrm{CDH})$ and of high frequency episodic headaches (HFEH) in a nationwide sample of Brazilian children and adolescents. Methods: This study was conducted as part of a large population study aiming to investigate the mental health of children and adolescents in Brazil (Attention-Brazil Project). The Attention-Brazil Project (ABP) was idealized by a free virtual academic community called Aprender Criança (www. aprendercrianca.com.br), dedicated to develop the interface between 
Neuroscience and Education. Of the approximately 4,500 members joining this community, 1, 151 teachers applied to participate in the ABP. Of them, 124 were selected and trained by internet (www.atencaobrasil.com.br) on how to select the sample and fill in the protocols. Of 8,304 children and adolescents randomly recruited at schools (target sample), consents and analyzable data were obtained from 6,383 (71.8\%) children and adolescents aged from 5 to 18 years $(49.8 \%$ girls), living in 87 cities and 18 Brazilian states, representing the all five national regions. Parents responded a validated questionnaire according to the Second Edition of the International Classification of Headache Disorders. The questionnaire is indeed the Portuguese version of the questionnaire used in the American Migraine Studies ${ }^{7}$ validated for use in the Brazilian population. ${ }^{8}$ The high frequency episodic headaches (HFEH) were defined as from 10-14 days of headache in the past month, and chronic daily headaches $(\mathrm{CDH})$ as 15 or more headaches in the past month. Crude and adjusted prevalence of $\mathrm{HFEH}$ and $\mathrm{CDH}$ were calculated. The level of significance adopted was 5\%. Statistical analysis was performed with the aid of the SPSS 15.0 for Windows (SPSS Inc.; Chicago IL). This study and surveys received full approval from a Human Research Committee and a written informed consents were obtained from all the parents. Results: The demographic of the final sample stratified by gender, age and race is showed in Table 1. The prevalence of chronic migraine was $0.6 \%$. The overall prevalence of $\mathrm{HFEH}$ was $1.5 \%$, significantly higher in girls $(1.9 \%$ vs. $1.1 \%, \mathrm{RR}=1.7,95 \% \mathrm{Cl}=1.1-2.6)$ and in adolescents older than 13 years $(2.8 \%$ vs. $1.0 \%$ in younger than 8 years-old, $R R=2.7, \mathrm{Cl}$ $=1.3-5.8)$. The prevalence of $\mathrm{CDH}$ was $1.0 \%$, significantly higher in adolescents older than 13 years $(2.2 \%$ vs. $0.7 \%$ in younger than 8 years-old, $\mathrm{RR}=3.4, \mathrm{Cl}=1.4-8.1)$ and in non-white $(1.4 \%$ vs. $0.8 \%$ in white ones, $\mathrm{RR}=1.8, \mathrm{Cl}=1.1-2.9)$. The overall prevalence of children and adolescents with 10 or more days of headache per month $(\mathrm{HFEH}+\mathrm{CDH})$ was $2.5 \%$, increasing with age $(5.0 \%$ in 14 18 years-old vs. $1.7 \%$ at age $5-7$ years-old, $R R=3.0,95 \% \mathrm{Cl}=$ 1.7-5.2) and significantly higher in girls (2.9\% vs. $2.1 \%$ in boys, RR $=1.4,95 \% \mathrm{Cl}=1.0-1.9, \mathrm{p}<0.05)$ (Table 2). Discussion: Frequent

Table 1. Demographic of the final sample stratified by gender, age and race

\begin{tabular}{|c|c|c|c|c|c|c|c|c|c|}
\hline \multirow[b]{2}{*}{ Age } & \multirow[b]{2}{*}{ Overall } & \multicolumn{4}{|c|}{ Female } & \multicolumn{4}{|c|}{ Male } \\
\hline & & White & Non-white & $\begin{array}{l}\text { Non } \\
\text { respondent }\end{array}$ & Total & White & Non-white & $\begin{array}{l}\text { Non } \\
\text { respondent }\end{array}$ & Total \\
\hline & $n(\%)$ & n (\%) & $\mathrm{n}(\%)$ & n (\%) & n (\%) & n (\%) & $n(\%)$ & n (\%) & n (\%) \\
\hline $5,6,7$ & $1984(31.1)$ & $683(34.4)$ & $217(10.9)$ & $57(2.9)$ & 957 (48.2) & $716(36.1)$ & $279(14.1)$ & $32(1.6)$ & $1027(51.8)$ \\
\hline $8,9,10$ & 3075 (48.2) & $1097(35.7)$ & $425(13.8)$ & $67(2.2)$ & 1589 (51.7) & $987(32.1)$ & $429(14.0)$ & $70(2.3)$ & $1486(48.3)$ \\
\hline $11,12,13$ & $962(15.1)$ & $288(29.9)$ & $150(15.6)$ & $12(1.2)$ & $450(46.8)$ & $310(32.2)$ & $182(18.9)$ & $20(2.1)$ & $512(53.2)$ \\
\hline $14,15,16+$ & $362(5.7)$ & $122(33.7)$ & $55(15.2)$ & $3(0.8)$ & $180(49.7)$ & $104(28.7)$ & $69(19.1)$ & $9(2.5)$ & $182(50.3)$ \\
\hline Overall & 6383 & 2190 & 847 & 139 & 3176 & 2117 & 959 & 131 & 3207 \\
\hline
\end{tabular}

Table 2. Prevalence of high frequency headache, chronic daily headaches and chronic migraine by age, gender, and race.

\begin{tabular}{|c|c|c|c|c|c|c|c|c|c|c|c|c|c|}
\hline \multirow[b]{2}{*}{ Age } & \multicolumn{2}{|c|}{ Overall } & \multicolumn{2}{|c|}{ Chronic Migraine } & \multicolumn{4}{|c|}{ HFEH } & \multicolumn{2}{|r|}{$\mathrm{CDH}$} & \multicolumn{3}{|c|}{$\mathrm{HFEH}+\mathrm{CDH}$} \\
\hline & $n$ & $\mathrm{n}$ & $\%$ & $\operatorname{RR}(95 \% \mathrm{Cl})$ & $n$ & $\%$ & $\operatorname{RR}(95 \% \mathrm{Cl})$ & $\mathrm{n}$ & $\%$ & $\operatorname{RR}(95 \% \mathrm{Cl})$ & $n$ & $\%$ & $\operatorname{RR}(95 \% \mathrm{Cl})$ \\
\hline $5,6,7$ & 1984 & 9 & 0,5 & reference & 20 & 1,0 & reference & 13 & 0,7 & reference & 33 & 1,7 & reference \\
\hline $8,9,10$ & 3075 & 19 & 0,6 & $1.4(0.6-3.0)$ & 49 & 1,6 & $1.6(0.9-2.6)$ & 32 & 1,0 & $1.6(0.8-3.0)$ & 81 & 2,6 & $1.6(1.1-2.4)$ \\
\hline $11,12,13$ & 962 & 6 & 0,6 & $1.4(0.5-3.8)$ & 15 & 1,6 & $1.5(0.8-3.0)$ & 12 & 1,2 & $1.9(0.9-4.2)$ & 27 & 2,8 & $1.9(1.0-2.8)$ \\
\hline $14,15,16+$ & 362 & 2 & 0,6 & $1.2(0.3-5.6)$ & 10 & 2,8 & $2.7(1.3-5.8)$ & 8 & 2,2 & $3.4(1.4-8.1)$ & 18 & 5,0 & $3.0(1.7-5.2)$ \\
\hline \multicolumn{14}{|l|}{ Gender } \\
\hline Female & 3176 & 20 & 0,6 & $1.3(0.6-2.4)$ & 59 & 1,9 & $1.7(1.1-2.6)$ & 33 & 1,0 & $1.0(0.6-1.7)$ & 92 & 2,9 & $1.4(1.0-1.9)$ \\
\hline Male & 3207 & 16 & 0,5 & reference & 35 & 1,1 & reference & 32 & 1,0 & reference & 67 & 2,1 & Reference \\
\hline \multicolumn{14}{|l|}{ Race } \\
\hline White & 4307 & 22 & 0,5 & reference & 62 & 1,4 & reference & 35 & 0,8 & reference & 97 & 2,3 & Reference \\
\hline Non-white & 1806 & 11 & 0,6 & $1.2(0.6-2.4)$ & 28 & 1,6 & $1.1(0.7-1.7)$ & 26 & 1,4 & $1.8(1.1-2.9)$ & 54 & 3,0 & $1.3(1.0-1.8)$ \\
\hline Non-respondents & 270 & 3 & 1,1 & $2.2(0.6-7.2)$ & 4 & 1,5 & $1.0(0.4-2.8)$ & 4 & 1,5 & $1.8(0.6-5.1)$ & 8 & 3,0 & $1.3(0.6-2.7)$ \\
\hline \multicolumn{14}{|l|}{ Income Class } \\
\hline$A, B$ & 2369 & 19 & 0,8 & $2.3(0.7-7.8)$ & 26 & 1,1 & reference & 34 & 1,4 & $1.8(0.8-4.0)$ & 60 & 2,5 & $1.0(0.6-1.6)$ \\
\hline$C$ & 3147 & 14 & 0,4 & $1.3(0.4-4.5)$ & 53 & 1,7 & $1.5(1.0-2.4)$ & 24 & 0,8 & $0.9(0.4-2.2)$ & 77 & 2,4 & $1.0(0.6-1.5)$ \\
\hline $\mathrm{D}, \mathrm{E}$ & 867 & 3 & 0,3 & reference & 15 & 1,7 & $1.6(0.8-3.0)$ & 7 & 0,8 & reference & 22 & 2,5 & Reference \\
\hline Total & 6383 & 36 & 0,6 & & 94 & 1,5 & & 65 & 1,0 & & 159 & 2,5 & \\
\hline
\end{tabular}

HFEH (high frequency episodic headaches): from 10-14 days of headache in the past month;

$\mathrm{CDH}$ (chronic daily headaches): 15 or more headaches in the past month. 
headaches are a significant problem in children and adolescents, often not effectively treated, in part because the scope of the problem remains poorly described, with consequent poor awareness. Most of the studies on the subject focused in adolescents or young adults, and established a prevalence of around $2 \%$ in adolescents, higher in girls. Populational and clinic-based studies also suggest that early onset of $\mathrm{CDH}$ is associated with poorer outcomes. Our most important findings were: 1) The prevalence of $\mathrm{CDH}$ and $\mathrm{HFEH}$ in Brazilian children and adolescents is relatively high, and important $2.5 \%$ have headaches on 10 or more days per month. 2) Adjusted prevalence was higher in girls than in boys and in non-white vs. white. 3) The prevalence markedly increased with age from preadolescent to adolescence age. 4) $\mathrm{CDH}$ and $\mathrm{HFEH}$ were seen in children as young as 5 years old. Our study has limitations. First, our questionnaire did not allow us to subclassify the headaches in specific headache syndromes (e.g. chronic migraine, chronic tensiontype headache). Follow-up investigations are addressing this topic. Nonetheless, regardless of the precise subtype, defining the prevalence of frequent headaches is of importance, and since this was a questionnaire-based study, we can't define if the headaches were primary or secondary. Second, we dichotomized race in White and non-White, which is certainly an over simplistic approach, since Brazil has a multiracial, miscegenated heritage. Nonetheless, the population surveyed by us consists basically of white, black, and brown individuals. In follow-up studies we intend to better explore the influence of race in primary headaches of pre-adolescent children. Strengths of our study include the population nature, robustness of a representative nationwide sample, use of well developed and validated questionnaires, and utilization of direct interviews (for applying the questionnaires). Frequent episodic headaches and chronic daily headaches are common in the pre-adolescent pediatric population. Future studies are necessary in order to identify risk factors for frequent headaches in the pediatric population, as well as impact of headaches on school and on social relationships. Based on our findings we estimate that 1.7 millions of children and adolescents in Brazil have 10 or more days of headache per month. Health care providers and educators should be aware of the magnitude of the problem, in order to properly identify and treat children with chronic headaches.

Disclosures: This study was conducted without commercial financial support. Dr Bigal is a full time employee of Merck Research Laboratories. He owns stocks and stock options of Merck. Merck did not sponsor this study. The study does not mention medications. Dr. Arruda has received grants from Janssen-Cilag for educational lectures on attention disorder deficit with hyperactivity. Renato Arruda report no disclosures.

A pesquisa foi aprovada por Comitê de Ética da Faculdade de Medicina de São José do Rio Preto.

\section{References:}

1. Koenig MA, Gladstein J, McCarter RJ, Hershey AD, Wasiewski W. Chronic daily headache in children and adolescents presenting to tertiary headache clinics. Headache 2002;42:491-500.

2. Bigal ME, Lipton RB, Tepper SJ, Rapoport AM, Sheftell FD. Primary chronic daily headache and its subtypes in adolescents and adults. Neurology 2004;63:843-847.

3. Seshia SS. Chronic daily headache in children and adolescents. Can J Neurol Sci 2004;31:319-323.

4. Chakravarty A. Chronic daily headache in children and adolescents: a clinic based study from India. Cephalalgia 2005;25:795-800.

5. Cuvellier JC, Couttenier F, Joriot-Chekaf S, Vallee L. Chronic daily headache in French children and adolescents. Pediatr Neurol 2008;38:93-98.

6. Arruda MA, Guidetti V, Galli F, Albuquerque RC, Bigal ME. Frequent headaches in the preadolescent pediatric population: a population-based study. Neurology 2010;74:903-908.

7. Lipton RB, Stewart WF, Simon D. Medical consultation for migraine: results from the American Migraine Study. Headache 1998;38:87-96.

8. Godoy DAG, Bigal ME, Jales LCF, Camparis CM, Speciali JG. Headache and Symptoms of Temporomandibular Disorder: an Epidemiological Study. Headache 2009 ; in press.

\title{
CE 21 - School achievement and absenteeism in school-aged children with primary headache - a Nationwide Study
}

\author{
Arruda MA'; Arruda R²; Bigal ME 3,4 \\ ${ }^{1}$ Director, Glia Institute, Ribeirao Preto, SP, Brazil; ${ }^{2}$ Graduate student, University of Campinas Medical School \\ ${ }^{3}$ Global Director for Scientific Affairs - Neuroscience, Merck Research Laboratories, Whitehouse Station, NJ, U.S. \\ ${ }^{4}$ Department of Neurology, Albert Einstein College of Medicine, Bronx, NY, U.S.
}

\begin{abstract}
Introduction: Headache is one of the most common symptoms in children and adolescents and headache syndromes respond to an important reason for medical consulting. A recent population study conducted by us showed a lifetime prevalence of headache in children and adolescents of $81 \%$. $^{1}$ Although migraine and tension-type headaches were the most common diagnoses, ${ }^{1}$ sizable $1.7 \%$ of the children had chronic daily headaches and $4.2 \%$ of the children had 10 or more days of headache by month. ${ }^{2}$ The burden of pediatric headaches has been well established as impacting the families and the children. The chronic headaches as a cause of school absenteism have been reported in the literature comparing children with chronic headache versus children without headache, ${ }^{3}$ children with migraine versus children without headache, and children with migraine versus children with tension-type headache. ${ }^{4}$
\end{abstract}

A positive correlation was found between the headache frequency and the numbers of school missing days. ${ }^{5}$ However, controversial findings are reported concerning the grade of impact of chronic headaches in school absenteism, ranging from $1 \%{ }^{6}$ to $68 \% .^{3}$ Very few studies investigated the impact of chronic headaches in cognitive functions in children and adolescents; however, to the best of our knowledge, studies assessing the impact of chronic headaches in school achievement have not been published. Accordingly, herein we take advantage of a nationwide populational study (Attention-Brazil Project) where children and adolescents were identified at schools, and parents and teachers were directly interviewed, in order to investigate whether chronic headaches impact school achievement and cause school absenteism. Methods: This study was conducted as part of a large population 
study aiming to investigate the mental health of children and adolescents in Brazil (Attention-Brazil Project). The Attention-Brazil Project (ABP) was idealized by a free virtual academic community called Aprender Criança (www.aprendercrianca.com.br), dedicated to develop the interface between Neuroscience and Education. Of the approximately 4,500 members joining this community, 1, 151 teachers applied to participate in the ABP. Of them, 124 were selected and trained by internet (www.atencaobrasil.com.br) on how to select the sample and fill in the protocols. Of 8,304 children and adolescents randomly recruited at schools (target sample), consents and analyzable data were obtained from 6,383 (71.8\%) children and adolescents aged from 5 to 18 years (49.8\% girls), living in 87 cities and 18 Brazilian states, representing the all five national regions. Parents responded a validated questionnaire according to the Second Edition of the International Classification of Headache Disorders. The questionnaire is indeed the Portuguese version of the questionnaire used in the American Migraine Studies ${ }^{7}$ validated for use in the Brazilian population. ${ }^{8}$ Based on the headache frequency, individuals were classified: a) no headache (in the past year); b) low frequency episodic headaches (LFEM, less than 5 days/month); c) intermediate frequency episodic headaches (IFEH, 5-9 days/month); d) high frequency episodic headaches (HFEH, 10-14 days/month); e) chronic daily headaches (CDH, 15 or more days/month). A validated questionnaire was responded by the parents and teachers to evaluate the school achievement, the frequency of headache in the classroom, the number of days the child was dismissed from school due to headache and, the number of missing school days due to headache in the last 6 months. Descriptive statistics were performed and relative risks (RR) were calculated to compare different groups. The level of significance adopted was $5 \%$. Statistical analysis was performed with the aid of the SPSS 15.0 for Windows (SPSS Inc.; Chicago IL). This study and surveys received full approval from a Human Research Committee and a written informed consents were obtained from all the parents. Results: Comparing to controls (no headache), children with $\mathrm{HFEH} / \mathrm{CDH}$ show a significantly lower school achievement $(R R=1.4,95 \% \mathrm{Cl}=1.1-1.7, p=0.009)$. No statistical significance is found comparing children with LFEH/IFEH, migraine and tension-type headache (TTH) to controls. Children with migraine show a significantly lower school achievement comparing to children with TTH $(R R=1.1,95 \% \mathrm{Cl}=1.0-1.3, \mathrm{p}=0.01)$, as well as children with $\mathrm{HFEH} / \mathrm{CDH}$ comparing to children with LFEH/ IFEH $(R R=1.3,95 \% \mathrm{Cl}=1.1-1.6, p=0.01)$ (Table 1). Children
Table 1. Prevalence and relative risk of 'below average' school achievement in children with tension-type headache, migraine, LFEH/IFEH and HFEH/CDH comparing to controls (no headache)

\begin{tabular}{cccccc}
\hline \multicolumn{5}{c}{ Below average' school achievement } \\
\hline & $\%$ & RR $(95 \% \mathrm{Cl})$ & $\mathrm{p}$ & $\mathrm{RR}(95 \% \mathrm{Cl})$ & $\mathrm{p}$ \\
\hline $\begin{array}{c}\text { No } \\
\text { headache } \\
\text { TTH }\end{array}$ & 28.0 & reference & - & - & - \\
Migraine & 30.2 & $0.9(0.8-1.0)$ & 0.30 & reference & - \\
LFEH/IFEH & 28.7 & $1.0(0.9-1.1)$ & 0.009 & reference & - \\
HFEH/CDH & 38.4 & $1.4(1.1-1.7)$ & 0.02 & $1.3(1.1-1.6)$ & 0.01 \\
\hline
\end{tabular}

TTH: tension-type headache; LFEH (low frequency episodic headache): less than 5 days of headache in the past month; IFEH (intermediate frequency episodic headache): from 5 to 9 days of headache in the past month; HFEH (high frequency episodic headaches): from 10-14 days of headache in the past month; $\mathrm{CDH}$ (chronic daily headaches): 15 or more headaches in the past month.

with migraine show a significantly higher frequency of headache in the classroom comparing to children with TTH in all endpoints: never, at least once, from 1 to 5 days, and more than 5 days, as well as children with HFEH/CDH comparing to children with LFEH/ IFEH (Table 2). Children with migraine more frequently was dismissed from school due to headache than children with TTH, as well as children with HFEH/CDH comparing to children with LFEH/ IFEH (Table 3). Finally, children with migraine show a significantly higher prevalence of absenteeism comparing to children with TTH in all endpoints: never $(69.3 \%$ vs. $75.7 \%, R R=0.9,95 \% \mathrm{Cl}=0.87$ $0.95, p<0.0001)$, at least once $(25.5 \%$ vs. $18.1 \%, R R=1.4,95 \%$ $\mathrm{Cl}=1.2-1.6, \mathrm{p}<0.0001)$, from 1 to 5 days $(10.3 \%$ vs. $6.1 \%$, $\mathrm{RR}=1.7,95 \% \mathrm{Cl}=1.3-2.1, \mathrm{p}<0.0001)$, and more than 5 days ( $1.5 \%$ vs. $0.7 \%, R R=2.0,95 \% \mathrm{Cl}=1.1-3.9, p=0.04)$, as well as children with HFEH/CDH comparing to children with LFEH/IFEH (Table 4). Conclusion: To the best of our knowledge, the present study is the first population-based study to investigate the school achievement in school-aged children with primary headache. Our findings show that HFEH/CDH impact school achievement and cause absenteeism comparing to LFEH/IFEH and controls (children and adolescents with no headache); and migraine impact school achievement and cause absenteism comparing to TTH. Bearing in

Table 2. Prevalence and relative risk of 'headache in the classroom' in children with migraine comparing to children with tension-type headache, and children with HFEH/CDH comparing to children with LFEH/IFEH.

\begin{tabular}{|c|c|c|c|c|c|c|c|c|c|c|c|c|}
\hline & \multicolumn{12}{|c|}{ Headache in the classroom in the last 6 months } \\
\hline & \multicolumn{3}{|c|}{ Never } & \multicolumn{3}{|c|}{ At least once } & \multicolumn{3}{|c|}{1 to 5 days } & \multicolumn{3}{|c|}{$>5$ days } \\
\hline & $\%$ & $\operatorname{RR}(95 \% \mathrm{Cl})$ & p & $\%$ & $\operatorname{RR}(95 \% \mathrm{Cl})$ & $p$ & $\%$ & $\operatorname{RR}(95 \% \mathrm{Cl})$ & p & $\%$ & $\operatorname{RR}(95 \% \mathrm{Cl})$ & p \\
\hline TTH & 63.0 & reference & & 32.3 & reference & & 10.8 & reference & & 1.5 & reference & \\
\hline Migraine & 57.2 & $0.9(0.8-0.9)$ & $<0.001$ & 38.5 & $1.2(1.1-1.3)$ & $<0.001$ & 14.4 & $1.3(1.1-1.6)$ & 0.001 & 3.7 & $2.4(1.5-3.6)$ & $<0.0001$ \\
\hline LFEH/IFEH & 62.2 & reference & & 32.9 & reference & & 12.0 & reference & & 2.1 & reference & \\
\hline $\mathrm{HFEH} / \mathrm{CDH}$ & 50.3 & $0.8(0.7-0.9)$ & 0.003 & 45.9 & $1.4(1.2-1.7)$ & 0.0009 & 22.6 & $1.9(1.4-2.5)$ & $<0.0001$ & 5.0 & $2.4(1.2-4.8)$ & 0.03 \\
\hline
\end{tabular}

TTH: tension-type headache; LFEH (low frequency episodic headache): less than 5 days of headache in the past month; IFEH (intermediate frequency episodic headache): from 5 to 9 days of headache in the past month; HFEH (high frequency episodic headaches): from 10-14 days of headache in the past month; $\mathrm{CDH}$ (chronic daily headaches): 15 or more headaches in the past month 
Table 3. Prevalence and relative risk of dismissing from school due to headache in children with migraine comparing to children with tension-type headache, and children with HFEH/CDH comparing to children with LFEH/IFEH

\begin{tabular}{|c|c|c|c|c|c|c|c|c|c|c|c|c|}
\hline & \multicolumn{12}{|c|}{ Dismissed from school due to headache in the last 6 months } \\
\hline & \multicolumn{3}{|c|}{ Never } & \multicolumn{3}{|c|}{ At least once } & \multicolumn{3}{|c|}{1 to 5 days } & \multicolumn{3}{|c|}{$>5$ days } \\
\hline & $\%$ & $\operatorname{RR}(95 \% \mathrm{Cl})$ & $\mathrm{p}$ & $\%$ & $\operatorname{RR}(95 \% \mathrm{Cl})$ & p & $\%$ & $\operatorname{RR}(95 \% \mathrm{Cl})$ & p & $\%$ & $\operatorname{RR}(95 \% \mathrm{Cl})$ & $\mathrm{p}$ \\
\hline TTH & 81.5 & reference & & 13.0 & reference & & 4.1 & reference & & 0.3 & Reference & \\
\hline Migraine & 76.4 & $0.9(0.9-1.0)$ & 0.0003 & 18.5 & $1.4(1.2-1.6)$ & $<0.0001$ & 6.0 & $1.5(1.1-1.9)$ & 0.01 & 0.4 & $1.3(0.4-3.9)$ & 0.82 \\
\hline LFEH/IFEH & 79.9 & reference & & 14.4 & reference & & 4.6 & reference & & 0.3 & Reference & \\
\hline $\mathrm{HFEH} / \mathrm{CDH}$ & 69.2 & $0.9(0.8-1.0)$ & 0.001 & 26.4 & $1.8(1.4-2.4)$ & $<0.0001$ & 7.5 & $1.6(0.9-2.9)$ & 0.12 & 1.9 & $6.0(1.8-20.5)$ & 0.009 \\
\hline
\end{tabular}

TTH: tension-type headache; LFEH (low frequency episodic headache): less than 5 days of headache in the past month; IFEH (intermediate frequency episodic headache): from 5 to 9 days of headache in the past month; HFEH (high frequency episodic headaches): from 10-14 days of headache in the past month; $\mathrm{CDH}$ (chronic daily headaches): 15 or more headaches in the past month.

Table 4. Prevalence and relative risk of missing school days due to headache in children with migraine comparing to children with tension-type headache, and children with HFEH/CDH comparing to children with LFEH/IFEH.

\begin{tabular}{|c|c|c|c|c|c|c|c|c|c|c|c|c|}
\hline & \multicolumn{12}{|c|}{ School days missed due to headache in the last 6 months } \\
\hline & \multicolumn{3}{|c|}{ Never } & \multicolumn{3}{|c|}{ At least once } & \multicolumn{3}{|c|}{1 to 5 days } & \multicolumn{3}{|c|}{$>5$ days } \\
\hline & $\%$ & $\operatorname{RR}(95 \% \mathrm{Cl})$ & $\mathrm{p}$ & $\%$ & $\operatorname{RR}(95 \% \mathrm{Cl})$ & $\mathrm{p}$ & $\%$ & $\operatorname{RR}(95 \% \mathrm{Cl})$ & $\mathrm{p}$ & $\%$ & $\operatorname{RR}(95 \% \mathrm{Cl})$ & $\mathrm{p}$ \\
\hline TTH & 75.7 & reference & & 18.1 & reference & & 6.1 & reference & & 0.7 & reference & \\
\hline Migraine & 69.3 & $0.9(0.87-0.95)$ & $<0.0001$ & 25.5 & $1.4(1.2-1.6)$ & $<0.0001$ & 10.3 & $1.7(1.3-2.1)$ & $<0.0001$ & 1.5 & $2.0(1.1-3.9)$ & 0.04 \\
\hline LFEH/IFEH & 74.2 & reference & & 19.7 & reference & & 7.6 & reference & & 0.9 & reference & \\
\hline $\mathrm{HFEH} / \mathrm{CDH}$ & 61.6 & $0.8(0.7-0.9)$ & 0.0006 & 34.0 & $1.7(1.4-2.2)$ & $<0.0001$ & 14.5 & $1.9(1.3-2.8)$ & 0.002 & 5.0 & $5.6(2.7-11.7)$ & $<0.0001$ \\
\hline
\end{tabular}

TTH: tension-type headache; LFEH (low frequency episodic headache): less than 5 days of headache in the past month; IFEH (intermediate frequency episodic headache): from 5 to 9 days of headache in the past month; HFEH (high frequency episodic headaches): from 10-14 days of headache in the past month; $\mathrm{CDH}$ (chronic daily headaches): 15 or more headaches in the past month

mind that $4.2 \%$ of Brazilian children and adolescents have 10 or more days of headache per month (2,77 millions of children) 2 ; $35.8 \%$ of them are dismissed from school due to headache at least one day per year $(991,000$ days), and 53.5\% miss school days due to headache at least one day per year (1,48 millions days), our data allow us to estimate that more than 2,4 millions of school days are missed per year in Brazil due to HFEH/CDH. Health care providers and educators should be aware of the magnitude of the problem, in order to properly identify and treat children with chronic headaches.

Disclosures: This study was conducted without commercial financial support. Dr Bigal is a full time employee of Merck Research Laboratories. He owns stocks and stock options of Merck. Merck did not sponsor this study. The study does not mention medications. Dr. Arruda has received grants from Janssen-Cilag for educational lectures on attention disorder deficit with hyperactivity. Renato Arruda report no disclosures.

A pesquisa foi aprovada por Comitê de Ética da Faculdade de Medicina de São José do Rio Preto.

\section{References:}

1. Arruda MA, Guidetti V, Galli F, Albuquerque RC, Bigal ME. Primary headaches in childhood - a population-based study. Cephalalgia : an international journal of headache 2010;30:1056-1064.

2. Arruda MA, Guidetti V, Galli F, Albuquerque RC, Bigal ME. Frequent headaches in the preadolescent pediatric population: a population-based study. Neurology 2010;74:903-908.

3. Abu-Arefeh I, Russell G. Prevalence of headache and migraine in schoolchildren. BMJ 1994;309:765-769.

4. Laurell K, Larsson B, Eeg-Olofsson O. Headache in schoolchildren: association with other pain, family history and psychosocial factors. Pain 2005;1 19:150-158.

5. Fichtel A, Larsson B. Psychosocial impact of headache and comorbidity with other pains among Swedish school adolescents. Headache 2002;42:766-775.

6. Collin C, Hockaday JM, Waters WE. Headache and school absence. Archives of disease in childhood 1985;60:245-247.

7. Lipton RB, Stewart WF, Simon D. Medical consultation for migraine: results from the American Migraine Study. Headache 1998;38:87-96.

8. Godoy DAG, Bigal ME, Jales LCF, Camparis CM, Speciali JG. Headache and Symptoms of Temporomandibular Disorder: an Epidemiological Study. Headache 2009 ;in press. 


\title{
CE 43 - Comparação entre o diagnóstico clínico da migrânea e o uso do ID-Migraine - Parte I
}

\author{
Jurno ME' ; Ferreira $\mathrm{AG}^{2}$; Mattos $\mathrm{AC}^{2}$; De Resende DF3; De Souza JA4; Moreira Filho PF 4 \\ 'Professor da FAME-FUNJOB e Coordenador da Residência do HRB-FHEMIG; ${ }^{2}$ Médicas Residentes; \\ ${ }^{3}$ Professor de Estatística da FAME-FUNJOB; ${ }^{4}$ Professores da Universidade Federal Fluminense
}

Introdução: A migrânea causa um grande impacto na saúde de seus portadores e na sociedade como um todo. Afeta por volta de $18 \%$ das mulheres e cerca de $6 \%$ dos homens durante toda sua vida útil, com pico de prevalência entre 25 e 55 anos de idade. ${ }^{1}$ Em estudos epidemiológicos populacionais a migrânea, especialmente com aura, foi associada a transtornos de ansiedade múltiplos, com conseqüente consumo exagerado de medicamentos abortivos. ${ }^{2}$ O diagnóstico impreciso e a falta de importância dos profissionais da saúde pelo tema pode ser um fator aditivo para estes pacientes demorar em ter um diagnóstico preciso e, consequentemente abusarem de medicação abortiva. ${ }^{3}$ Desta forma, a migrânea tem sido relatada como uma das 20 patologias que mais causam desabilidade na vida de seus portadores, sendo responsável por $1,4 \%$ de todas as causas de desconforto. ${ }^{4}$ Estabelecer o diagnóstico correto é essencial para o sucesso terapêutico. $\bigcirc$ diagnóstico deve ser realizado através das características clínicas da dor, do comportamento de indivíduo e de fatores associados, não havendo um marcador biológico específico para a migrânea. ${ }^{5}$ Objetivo: O objetivo deste estudo foi fazer a comparação do diagnóstico de pacientes migranosos atendidos no ambulatório de cefaleia através da aplicação de uma entrevista estruturada: ID-Migraine ${ }^{4}$ e compará-lo aos critérios diagnósticos descritos na Classificação Internacional das Cefaleias. ${ }^{6}$ Material e métodos: Esta pesquisa foi um estudo de corte transversal, com análise comparativa do diagnóstico de um grupo de pacientes com dor de cabeça, através de duas ferramentas distintas - Migraine-ID e consulta neurológica, respeitando os critérios elencados na Classificação Internacional das Cefaléias. ${ }^{7}$ Em uma abordagem inicial, aqueles pacientes que estavam na sala de espera, agendados para ser atendido nos ambulatórios de dor de cabeça do Hospital Regional de Barbacena (HRB/FHEMIG) e da Universidade Federal Fluminense (UFF), foram convidados a participar do experimento e assinarão o termo de consentimento livre e esclarecido. Para aqueles que concordaram em participar da pesquisa foram apresentadas as perguntas propostas pelo Migraine-ID ${ }^{4}$ : a) Sentiu-se nauseada(o) indisposta(o) enquanto estava com dor de cabeça? ; b) A luz incomodou-a(o) (muito mais do que quando não tem dor de cabeça)?; c) As suas dores de cabeça limitaram a sua capacidade de trabalhar, estudar, ou fazer o que precisava de fazer durante, pelo menos, um dia? $\bigcirc$ diagnóstico de migrânea foi firmado com resposta positiva às três questões. Após a consulta com o neurologista deste ambulatório, onde foram utilizados os critérios de referência da Classificação Internacional das Cefaleias o resultado do Migraine-ID foi comparado ao diagnóstico firmado pelo médico neurologista. A análise dos dados foi feita em microcomputadores, com recursos de processamento estatístico do "software" Stata 9.2. As variáveis da análise foram as registradas nos questionários do projeto. Foi determinada a concordância do diagnóstico de migrânea detectáveis entre os pacientes que se dispuseram em participar do experimento, além de serem construídas as distribuições de frequências e calculados outros percentuais, médias e desvios padrões interesse do estudo. A concordância entre os diagnósticos clínico e o fornecido pela Migrânea-ID foi avaliada através do coeficiente Kappa. $\bigcirc$ nível de significância foi estabelecido em 0,05. Foram também calculadas as taxas de sensibilidade e especificidade do Migrânea-ID, tomando as taxas de positivos e negativos para a presença de migrânea, obtidas com e sem diagnóstico firmado pelo neurologista, tendose como referência a Classificação Internacional das Cefaléias. ${ }^{9}$ Resultados: Nesta primeira parte deste estudo foram avaliados 320 pacientes cujas idades variaram de 18 a 64 anos (media de $38,3 \pm 11,5$ anos). Da amostra, 275 pacientes $(85,9 \%)$ eram do sexo feminino. Foram incluídos na pesquisa todos os pacientes que apresentavam queixa de cefaleia e os diagnósticos mais frequentes foram o seguinte: Migrânea sem aura 65,94\%; migrânea com aura 6,25\%; cefaleia tipo tensional frequente 15\%; cefaleia por abuso de medicamentos 10\%. Após a resposta ao questionamento do Migrânea-ID e consulta neurológica, a concordância com o diagnóstico clinico para migrânea sem aura foi de 70,14\% e para migrânea com aura foi de 90,0\%. Conclusões: Os resultados obtidos nesta pesquisa demonstraram que o questionário do Migrânea-ID pode ser uma ferramenta útil no screening para o auxílio no diagnóstico da migrânea e os achados nesta primeira parte de nossa pesquisa encontraram valores próximos aos dados presentes na literatura.

Referências:

1) Lipton RB, Stewart WF, von Korff M. Burden of migraine: Societal costs and therapeutic opportunities. Neurology. 1997; 48(Suppl 3):S4-S9.

2) Mitsikostas DD, Thomas AM. Comorbidity of headache and depressive disorders. Cephalalgia. 1999; 19:211-7.

3) Diener HC, Limmroth V. Medication-overuse headache: a worldwide problem. Lancet Neurol .2004; 3: 475-83.

4) Gil-Gouveia R, Martinsl. Validation of the Portuguese Version of ID-Migraine. Headache. 2010; 50:396-402.

5) Joubert J. Australian Family Phisician. August 2003; 34(8):627-32.

6) Subcomitê de Classificação das Cefaléias. Classificação Internacional das Cefaléias. $2^{a}$ Ed. Tradução da Sociedade Brasileira de Cefaléia. São Paulo. Alaúde editorial. 2006. 286p.

7) Lipton RB, Dodick D, Sadovsky R et al. A self-administtered secreener for migraine in primary care - The ID Migraine validation study. Neurology. 2003;61:375-382. 


\title{
CE 45 - Cefaleia em salvas: avaliação epidemiológica brasileira - estudo de 387 casos
}

\author{
Nobre ME, Moreira Filho PF \\ Rio de Janeiro
}

Introdução e objetivo: A cefaleia em salvas (CS), seguramente a mais intrigante das cefaleias, é rara e apresenta características clínicas peculiares. O estudo epidemiológico mais recente, realizado por Goadsby em 2002, trouxe à reflexão conceitos epidemiológicos já estabelecidos, sugerindo ser uma dor mais comum do que antes se conhecia. Observou-se também a demora diagnóstica pela falta de familiaridade dos médicos com os critérios diagnósticos. Além disso, novas características clínicas têm sido observadas e descritas. $\bigcirc$ nosso objetivo é realizar uma avaliação epidemiológica nacional visando contribuir para o aprimoramento dos critérios diagnósticos já existentes. Método: Pacientes portadores de CS, já com diagnóstico estabelecido, preencheram um questionário online. Foram avaliados 658 questionários entre janeiro de 2009 e dezembro de 2010 . Destes, foram selecionados 387. Os indivíduos foram entrevistados por telefone e em consulta presencial, quando possível, para confirmação do diagnóstico de acordo com os critérios da Sociedade Internacional de Cefaleia. Foram excluídos os pacientes com outros tipos de cefaleias primárias. Os seguintes itens foram estudados: sexo, raça, idade no atendimento, cor dos olhos, diagnóstico inicial, padrão da dor, horário, lateralidade da dor, sinais e sintomas, intensidade da dor, localização, presença de tabagismo e etilismo, presença de história familiar, comorbidades, exames complementares realizados, índice de massa corpórea e presença de ronco. Resultados: Serão apresentados os resultados da análise estatística de itens selecionados. Houve predomínio do sexo masculino, com 283 indivíduos (73.1\%) e 104 (26.9\%) do sexo feminino, evidenciando uma relação de 2,7:1. A média de idade foi de 39.3 anos no momento da entrevista. A raça branca foi predominante (320 - 82,7\%) e a cor dos olhos mais encontrada foi castanho escuro (275 -71,1\%). $\bigcirc$ primeiro diagnóstico foi cefaleia em salvas em apenas 111 (28,7\%) dos indivíduos. $\bigcirc$ padrão mais encontrado foi a forma episódica, com $259(66,9 \%)$ com a forma direta e $17(4,4 \%)$ com a forma episódica originada de crônica, portanto $276(71,3 \%)$ no total. Em relação à lateralidade, observou-se dor à direita em $189(48,8 \%)$ indivíduos, esquerda em 150 (38,8\%), alternância de lado na mesma salva em 11 (2,8\%), alternância de lado em salvas diferentes em 32 (8,3\%) e bilateral em $5(1,3 \%)$ indivíduos. A dor foi excruciante em 73,9\% (286) dos casos e o lacrimejamento foi o sintoma mais frequente (327-84,5\%). O horário onde houve maior incidência de crises foi entre 0 e $2 \mathrm{~h}$. A incidência de tabagismo foi de 55,8 dos pacientes e uso de álcool pelo menos duas vezes por semana foi de $56,1 \%$. Conclusões: Observamos um predomínio do sexo masculino, mas uma relação menor que a referida na literatura. Houve uma incidência maior de casos crônicos. A dor não se mostrou estritamente unilateral, o que já havia sido relatado na literatura, porém observamos uma incidência maior. A demora no diagnóstico é uma característica mundial, mas com esse estudo podemos observar que devemos investir na educação médica continuada para reduzir o tempo de sofrimento destes pacientes.

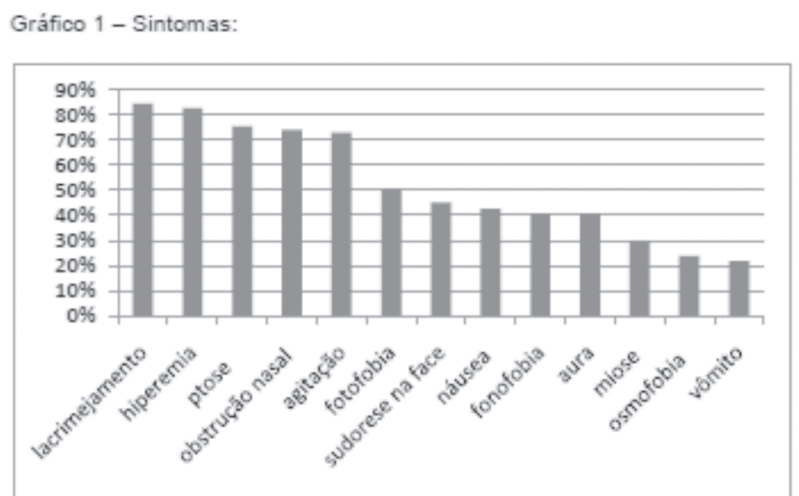

Gráfico demonstrando a incidência dos sinais e sintomas em ordem decrescente

Gráfico 2: Horários de maior incidência:

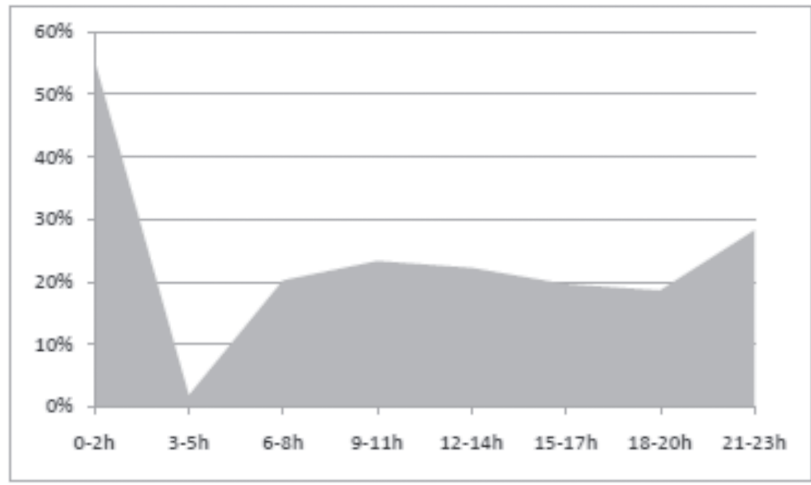

Bibliografia:

1. Broner S W, Cohen J M. Epidemiology of Cluster Headache. Current Pain and Headache Reports 2009; 13:141-146.

2. Fischera M, Marziniak M, Gralow I, Evers S. The incidence and prevalence of cluster headache: a meta-analysis of population-based studies. Cephalalgia, 2008; 28: 614-618.

3. Bahra A, May A, Goadsby P J. Cluster headache - a prospective clinical study with diagnostic implications. Neurology, 2002; 58:354-361.

4. Manzoni G C, Terzano M G, Bono G, Micieli G, Martucci N, Nappi G. Cluster headache - clinical findings in 180 patients. Cephalalgia, 1983; 3: 21-30. 


\title{
CE 57 - Cefaleia na instalação do acidente vascular cere- bral: Uma análise de 3.550 casos
}

\author{
Carvalho JJF; Machado CB; Viana GAA; Lemos ACS; Alves MB; Santos BC; Silva GS \\ Secretaria de Saúde do Estado do Ceará / Hospital Albert Einstein
}

\begin{abstract}
Introdução: Cefaleia é o motivo de consulta de 2 a $4 \%$ das pessoas que procuram uma emergência. Em muitos casos a dor de cabeça acontece no contexto de um Acidente Vascular Cerebral (AVC). Reconhecida desde 1664 por Thomas Willis, a relação entre cefaleia e doença cerebrovascular, somente no século passado teve o primeiro estudo abrangente publicado por Miller Fisher.' Desde então, vários trabalhos apontam para uma frequência entre 7 e $65 \%$ o que reflete a variedade de denominações e metodologias. ${ }^{2} \mathrm{Na}$ instalação, estima-se que entre 17 e 34\% dos pacientes apresentem dor de cabeça. ${ }^{3}$ Esta frequência pode estar subestimada, pois muitos pacientes apresentam algum grau de comprometimento da consciência que associado a alterações de linguagem e do estado mental frequentemente inviabilizam uma boa anamnese. A despeito destas observações, muitos aspectos ainda persistem controversos, dentre eles, um meIhor entendimento da cefaleia associada à instalação do AVC. Ob-
\end{abstract} jetivo: Este trabalho, objetiva estudar a frequência e fatores associados à cefaleia como sintoma inaugural de AVC em uma grande população de pacientes. Método: Analisamos as fichas de 3.550 pacientes investigados no projeto de vigilância epidemiológica do AVC em Fortaleza desenvolvido desde junho de 2009 pela Secretaria de Saúde do Estado do Ceará, com o apoio e suporte do Instituto de Responsabilidade Social do Hospital Israelita Albert Einstein de São Paulo. Este trabalho foi aprovado pelo Comitê de ética do Hospital Geral de Fortaleza. Baseadas no programa Steps Stroke proposto pela Organização Mundial da Saúde, as fichas, além dos dados demográficos contém o estado funcional anterior ao AVC, os sintomas inaugurais, os fatores de risco e dados da hospitalização, diagnóstico, exames complementares, tratamento multiprofissional, complicações, evolução e desfecho. A presença de cefaleia na instalação do AVC foi considerada quando referida, sob questionário, pelo paciente ou familiar ou acompanhante que o assistiu quando da instalação do quadro. Os dados foram tratados estatisticamente com testes não-paramétricos (teste do Qui-quadrado) além de frequências absolutas e porcentagens. Em todos os casos foram considerados significantes resultados com $p=0,05$. Resultados: Foram 1854 (52\%) mulheres e 1696 (48\%) homens, com idade média de $67 \pm 14$ anos com 2540 (72\%) AVC's isquêmicos (AVCI), 508 (14\%) AVC's hemorrágicos (AVCH), 121 (3\%) hemorragias subaracnoideas (HSA), 104 (3\%) ataques isquêmicos transitórios (AIT) e 277 (8\%) AVC's não especificados (se isquêmicos ou hemorrágicos). Em 213 pacientes, não foi possível confirmar ou não a presença de cefaleia na instalação do AVC. Nos demais pacientes, a cefaleia esteve entre os sintomas inaugurais do AVC em 30,8\% dos casos. Quanto ao tipo de AVC ela esteve presente em $23 \%$ dos AlT's, $26 \%$ dos $\mathrm{AVCl}^{\prime}$ s, $44 \%$ dos $\mathrm{AVCH}$ 's e $84 \%$ das HSA's. As mulheres relataram mais dores de cabeça que os homens $(33 \% \times 29 \%, p=0,0282)$ assim como os pacientes mais jovens (abaixo dos 50 anos) (55\% x 27\%, p < 0,0001). Não foram observadas diferenças quanto à raça. Em relação aos fatores de risco, pacientes com hipertensão arterial (29\% x $41 \%, p<0,0001)$, diabetes (25\% x 35\%, p < 0,0001), AVC prévio (27\% x 35\%, p < 0,0001) e Fibrilação Atrial $(25 \% \times 32 \%, p=0,0070)$ apresentaram menos dores de cabeça, porém, pacientes tabagistas $(36 \%$ × 30\%, $p=0,0022)$ e etilistas $(35 \% \times 31 \%, p=0,0215)$ referiram o sintoma de forma muito mais frequente. Cefaleia foi sintoma isolado na apresentação de 3\% dos AVCH's e 16\% nos casos de HSA. Quanto ao desfecho, a presença de cefaleia na instalação se correlaciona com menor taxa de óbito (27 \% x 32\%, p = 0330) (Tabela 1). Discussão: Ainda não há consenso sobre uma definição e mecanismo fisiopatológico da cefaleia que ocorre na instalação do AVC. Na literatura, por exemplo, encontram-se diversas definições temporais. Alguns estudos definem como uma dor que ocorre de 48 horas antes até 24 horas depois do AVC. Outros adotam um período de 3 dias antes e depois do ictus e outros ainda não estabelecem prazos. ${ }^{3} \mathrm{Em}$ nosso estudo, adotamos o critério de cefaleia que se apresentou em clara relação temporal e, definitivamente, fez parte da constelação de sintomas que levaram o paciente a procurar auxílio médico que resultou no diagnóstico do primeiro ou um novo AVC. Como já relatado, os nossos pacientes mais jovens e as do sexo feminino apresentaram mais dores de cabeça. Também de acordo com outros trabalhos, o nossos pacientes com hipertensão arterial apresentaram menos cefaleias. Em nossa casuística, porém, a presença de diabetes, AVC prévio e fibrilação atrial também estiveram relacionados com uma menor incidência do sintoma. Por outro lado, o tabagismo e o etilismo se correlacionaram com mais dor. Infelizmente, não temos informações detalhadas das características das cefaleias a permitir a classificação das mesmas, nem detalhes dos exames de neuroimagem que possibilitariam tipificar melhor os AVC's quanto ao tamanho, regiões cerebrais e territórios vasculares acometidos. Certamente isso nos permitiria uma melhor correlação dos achados. Não temos ainda um modelo acabado que reflita os mecanismos envolvidos na cefaleia associada à instalação da doença cerebrovascular. Várias conjecturas são elencadas nos diversos trabalhos sobre o tema, todas no terreno das hipóteses. ${ }^{4,5,6}$ Certamente, desde mecanismos reflexos que podem induzir dor a partir do território lesado à participação do sistema trigêmino-vascular, dos sistemas simpático, parassimpático e neuroendócrino além de mediadores locais, são muitas e, possivelmente, multifatoriais as possiblidades fisiopatológicas. Conclusão: Nossos dados demonstram que a cefaleia, presente na instalação de cerca de um terços dos AVC's sinaliza para a etiologia hemorrágica (AVCH e HSA) e menor taxa de óbitos hospitalares por determinantes que ainda precisam ser melhor estabelecidos em estudos apropriados.

Referências:

1. Arboix A, Massons J, Oliveres M, Arribas MP, Titus F. Headache in acute cerebrovascular disease: a prospective clinical study in 240 patients. Cephalalgia 1994; 14:37-40.

2. Carolei A, Sacco S. Headache attributed to stroke, TIA, intracerebral haemorrhage, or vascular malformation. Handb Clin Neurol. 2010; 97:517-28.

3. Mitsias PD, Ramadan NM, Levine SR, Schultz L \& Welch KMA. Factors determining headache at onset of acute ischemic stroke. Cephalalgia 2006; 26:150-157.

4. Kumral E, Bogousslavsky J, Van Melle G et al. Headache at stroke onset: the Lausanne Stroke Registry. J Neurol Neurosurg Psychiatry 1995; 58: 490-492.

5. Tentschert S, Wimmer R, Greisenegger S et al. Headache at stroke onset in 2196 patients with ischemic stroke or transient ischemic attack. Stroke 2005; 36: e 1 -e3.

6. Verdelho A, Ferro JM, Melo T, Canhão P \& Falcão F. Headache in acute stroke. A prospective study in the first 8 days. Cephalalgia 2008; 28:346-354. 


\title{
DOR 08 - Fibromialgia em pacientes com disfunção temporomandibular: prevalência e estudo comparativo do limiar de dor à pressão
}

\author{
Stuginski-Barbosa J'; Murayama RA²; Moraes NP3 \\ 'Cirurgiã-dentista, especialista em DTM e Dor Orofacial, Mestre em Neurociências pela Faculdade de Medicina de \\ Ribeirão Preto (USP), aluna de doutorado em Reabilitação Oral pela Faculdade de Odontologia de Bauru (USP) \\ ${ }^{2}$ Cirurgião-dentista, especialista em DTM e Dor Orofacial, Mestre e Doutor em Estomatologia pela Faculdade de \\ Odontologia de Araçatuba (UNESP), graduando em Medicina pelo Centro Universitário São Camilo \\ ${ }^{3}$ Cirurgião-dentista, professor titular da Faculdade de Odontologia de Araçatuba (UNESP)
}

Estudos epidemiológicos indicam uma taxa significativa de presença de pessoas apresentando dor crônica maior do que 46,5\% entre a população em geral. Este fato pode contribuir para o aumento na demanda por tratamento de enfermidades caracterizadas pela presença de dor crônica, o que inclui a disfunção temporomandibular (DTM) e a fibromialgia (FM). Essas entidades patológicas podem causar grande impacto na qualidade de vida, incluindo taxas de depressão, ansiedade e até mesmo mortalidade, tornando a identificação dos grupos de risco para este tipo de dor extremamente importante. Objetivos: Determinar a prevalência de FM em pacientes com DTM e comparar o limiar de dor à pressão (LDP) em músculos mastigatórios de pacientes com FM associada ou não a DTM (dor miofascial) e indivíduos assintomáticos. Métodos: A prevalência de FM foi avaliada em 274 pacientes com DTM. Entre os pacientes com DTM, quando havia suspeita de FM a partir de dados da história médica, questionários específicos (ANEXO C), e presença de dor pelo corpo em pelo menos 11 pontos bilaterais, o paciente era encaminhado ao médico reumatologista para diagnóstico de FM segundo os critérios da Academia Americana de Reumatologia (Wolfe et al., 1990). Foram encaminhados 39 pacientes e confirmados 29 casos que foram submetidos à aplicação dos seguintes critérios de exclusão para compor o grupo FM e DTM: ausência de 5 ou mais dentes posteriores em paciente não reabilitado por prótese, presença de doenças sistêmicas não controladas como controladas como hipotireoidismo, artrite reumatóide e anemia perniciosa entre outras. Foram então selecionados 15 pacientes. Para comparação do LDP, foram selecionados mais 42 indivíduos do sexo feminino. Foram formados quatro grupos: grupo FM e DTM $(n=15)$, grupo DTM $(n=15)$, grupo FM $(n=12)$ e grupo controle $(n=15)$. Indivíduos com FM não poderiam apresentar dor orofacial nos últimos 3 meses. Indivíduos do grupo controle não poderiam apresentar dor no corpo ou orofacial nos últimos 3 meses. Avaliou-se o LDP em pontos localizados em músculos masseter, temporal bilateral e ponto controle no leito ungueal do dedo polegar direito por algometria de pressão. Os dados obtidos foram analisados por teste ANOVA, pós teste de Tukey, com nível de significância de 5\% e intervalo de confiança de 95\%. O diagnóstico para DTM seguiu os critérios do Research Diagnostic Criteria for Temporomandibular Disorders e para FM os critérios da Academia Americana de Reumatologia (ACR). A pesquisa foi aprovada pelo Comitê de Ética e Pesquisa em Humanos sob o protocolo 2006-01459. Resultados: 29 pacientes com DTM preencheram critérios para FM (10,58\%). $\bigcirc$ LDP mostrou ser sempre maior no grupo controle, seguido em ordem decrescente pelo grupo DTM, FM e FM+DTM. Houve diferença significativa entre o grupo controle e os demais grupos no ponto controle e músculos masseter e temporal esquerdo $(p<0,001)$ e entre o grupo controle e o grupo FM+DTM em masseter e temporal direito. Não houve diferenças entre os grupos DTM, FM e FM+DTM.A diminuição do LDP em ponto controle nos indivíduos com SFM, DTM e SFM+DTM, em relação aos indivíduos saudáveis, demonstra indício de sensibilização central. A utilização do leito ungueal como ponto controle mostrou ser de fácil acesso para realização da algometria. Conclusões: Os dados sugerem que pacientes com DTM e/ou FM apresentam diferenças com relação ao processamento nociceptivo quando comparados à indivíduos saudáveis, com aumento na sensibilidade dolorosa. $\bigcirc$ fato de ocorrer diferenças em áreas extracefálicas sugere a presença de sensibilização central, sobretudo em pacientes com as duas condições. A FM e a DTM podem compartilhar de passos fisiopatológicos semelhantes e possuir correlação e continuidade temporal e surgir simultaneamente ou proceder uma a outra em uma associação mais do que casual. A presença de FM deve ser investigada em pacientes com DTM.

Tabela I - Resultados da algometria com relação ao Limiar de dor à pressão obtida em músculos da mastigação e ponto controle em $\mathrm{kg} / \mathrm{cm}^{2} / \mathrm{seg}$ a partir dos grupos estudados

\begin{tabular}{|c|c|c|c|c|}
\hline Pontos & $\begin{array}{c}\text { Grupo } \\
\text { FM+DTM } \\
(n=15)\end{array}$ & $\begin{array}{c}\text { Grupo } \\
\text { FM } \\
(n=15)\end{array}$ & $\begin{array}{c}\text { Grupo } \\
\text { DTM } \\
(n=13)\end{array}$ & $\begin{array}{l}\text { Grupo } \\
\text { controle } \\
(\mathrm{n}=15)\end{array}$ \\
\hline $\begin{array}{l}\text { Ponto controle } \\
\text { Média } \\
\text { Desvio padrão } \\
\text { Mediana }\end{array}$ & $\begin{array}{l}2,22 \\
0,97 \\
2,10\end{array}$ & $\begin{array}{l}2,41 \\
0,86 \\
2,33\end{array}$ & $\begin{array}{l}2,85 \\
1,28 \\
2,8\end{array}$ & $\begin{array}{l}4,67 \\
2,08 \\
4,03\end{array}$ \\
\hline $\begin{array}{l}\text { Masseter direito } \\
\text { Média } \\
\text { Desvio padrão } \\
\text { Mediana }\end{array}$ & $\begin{array}{l}1,16 \\
0,50 \\
1,14\end{array}$ & $\begin{array}{l}1,32 \\
0,34 \\
1,32\end{array}$ & $\begin{array}{l}1,40 \\
0,37 \\
1,38\end{array}$ & $\begin{array}{l}1,70 \\
0,47 \\
1,71\end{array}$ \\
\hline $\begin{array}{l}\text { Masseter esquerdo } \\
\text { Média } \\
\text { Desvio padrão } \\
\text { Mediana }\end{array}$ & $\begin{array}{l}1,04 \\
0,23 \\
1,04\end{array}$ & $\begin{array}{l}1,29 \\
0,37 \\
1,38\end{array}$ & $\begin{array}{r}1,23 \\
0,32 \\
1,21\end{array}$ & $\begin{array}{l}1,83 \\
0,42 \\
1,77\end{array}$ \\
\hline $\begin{array}{l}\text { Temporal anterior direito } \\
\text { Média } \\
\text { Desvio padrão } \\
\text { Mediana }\end{array}$ & $\begin{array}{l}1,10 \\
0,48 \\
0,96\end{array}$ & $\begin{array}{l}1,29 \\
0,29 \\
1,18\end{array}$ & $\begin{array}{l}1,39 \\
0,44 \\
1,43\end{array}$ & $\begin{array}{l}1,70 \\
1,69 \\
1,70\end{array}$ \\
\hline $\begin{array}{l}\text { Temporal anterior esquerdo } \\
\text { Média } \\
\text { Desvio padrão } \\
\text { Mediana }\end{array}$ & $\begin{array}{l}1,11 \\
0,48 \\
1,20\end{array}$ & $\begin{array}{l}1,30 \\
0,36 \\
1,28\end{array}$ & $\begin{array}{l}1,40 \\
0,39 \\
1,36\end{array}$ & $\begin{array}{l}1,93 \\
0,51 \\
1,93\end{array}$ \\
\hline
\end{tabular}




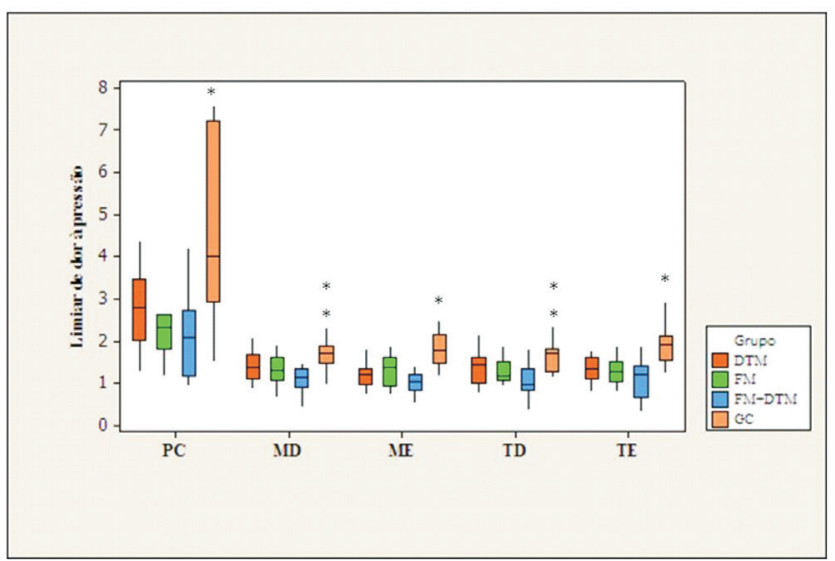

Referências

Dworkin SF, LeResche L. Research diagnostic criteria for temporomandibular disorders: review, criteria, examinations and specifications, critique. J Craniomandib Disord. 1992;6(4):301-55

Gracely RH, Grant MAB, Giesecke T. Evoked pain measures in fibromyalgia. Best Pract Res Clin Rheumatol 2003: 17(4): 593-609.

Julien N, Goffaux P, Arsenault P, Marchand S. Widespread pain in fibromyalgia to a deficit of endogenous pain inhibition. Pain 2005;1 14: 295-302.

Michelloti A, Farella M, Stellato A, Martina R. Tactile and pain thresholds in patients with miofascial pain of the jaw muscles: a case-control study. J Orofacial Pain 2008;22:139-45.
Gráfico 1 - Comparação entre os grupos Disfunção Temporomandibular (DTM), Fibromialgia (FM), DTM e FM e grupo controle (GC) com relação ao limiar de dor à pressão nos diferentes pontos avaliados.

PC - Ponto controle; MD - Masseter Direito; ME - Masseter Esquerdo; TD - Temporal Direito; TE - Temporal Esquerdo; * diferença significativa na comparação entre o grupo controle e os demais grupos; ${ }^{* *}$ diferença significativa na comparação entre os grupos controle e FM+DTM, por teste ANOVA, pós-teste de Tukey

Santos Silva R, Conti PC, Lauris JR, da Silva RO, Pegoraro LF. Pressure pain threshold in the detection of masticatory myofascial pain: an algometer-based study. J Orofacial Pain 2005; 19(4):318-324.

Staud R, Smitherman ML. Peripheral and central sensitization in fibromyalgia: pathogenetic role. Current Pain and Headache Reports 2002;6:259-266.

Wolfe F, Smythe HA, Yunus MB, Bennett RM, Bombardier C, Goldenberg DL, et al. The American College of Rheumatology 1990 Criteria for the Classification of Fibromyalgia. Report of the Multicenter Criteria Committee. Arthritis Rheum. 1990;33(2): 160-72

\title{
DOR 12 - Temporomandibular disorder pain and sleep bruxism increase risk for primary headache Disfunção temporomandibular dolorosa e bruxismo do sono aumentam o risco para cefaleia primária
}

\author{
Fernandes $G^{1}$, Franco $\mathrm{AL}^{2}$, Speciali $\mathrm{JG}^{3}$, Bigal $\mathrm{ME}^{4}$, Gonçalves $\mathrm{DAG}^{5}$, Camparis $\mathrm{CM}^{6}$ \\ ${ }^{1,2}$ Aluna de Pós-Graduação, ${ }^{3}$ Professor Associado. Depto. de Neurologia, Faculdade de Medicina de Ribeirão Preto, USP, \\ São Paulo, Brasil, ${ }^{4}$ Chefe do Laboratório de Pesquisa e do Grupo de Educação Científica da Merck - Merck Laboratórios, \\ Depto. de Neurologia, Faculdade Albert Einstein de Medicina, Bronx, NY. \\ ${ }^{5}$ Professora Assistente, ${ }^{6}$ Professora Adjunto. Disciplina de Oclusão, Desordens Temporomandibulares e \\ Dores Orofaciais, Depto. de Materiais Odontológicos e Prótese, Fac.de Odontologia de Araraquara, UNESP, São Paulo, Brasil
}

Introdução: A Academia Americana de Medicina do Sono (AAMS)' define o Bruxismo do Sono (BS) como uma atividade oral caracterizada pelo ranger ou apertar dos dentes durante o sono. Disfunção Temporomandibular (DTM) é um termo coletivo que abrange problemas clínicos envolvendo a musculatura da mastigação, a articulação temporomandibular (ATM) e estruturas associadas. ${ }^{2}$ Apesar das dificuldades metodológicas para a obtenção de conclusões definitivas, o BS pode ser considerado um importante fator de iniciação e perpetuação da DTM dolorosa. ${ }^{3}$ As DTMs e as Cefaleias Primárias também parecem estar associadas, sendo consideradas entidades distintas, atuando uma sobre a outra como fator perpetuante e agravante. ${ }^{4}$ Finalmente, as cefaleias e o bruxismo do sono também parecem estar associados, ${ }^{5}$ porém ainda não há nenhum estudo a respeito da associação múltipla entre BS, DTM e cefaleias primárias. Proposição: Investigar uma possível associação entre essas três entidades, avaliando o risco de ocorrência de cefaleias primárias em pacientes com ou sem DTM dolorosa e BS. Material e Método: A amostra foi composta por indivíduos de ambos os gêneros, com idade entre 18 a 76 anos, que procuraram tratamento na clínica de DTM e Dor Orofacial da Faculdade de Odontologia de Araraquara - UNESP. Os critérios de exclusão foram a presença de odontalgia, dor neuropática, presença de DTM por menos de 3 meses, lesões intra-orais, síndromes dolorosas crônicas. Todos os indivíduos foram submetidos a um protocolo de diagnóstico sistematizado, realizado por um examinador calibrado:

a) Ficha Clínica de DTM e Dor Orofacial da FOAr - UNESP para detalhar a queixa principal, as características da dor e permitir o diagnóstico diferencial de condições que pudessem mimetizar as DTMs2.

b) Research Diagnostic Criteria for Temporomandibular Disorder (RDC/TMD), para confirmação do diagnóstico e classificação da DTM.

c) Questionário para diagnóstico inicial das cefaleias primárias baseado nos critérios propostos pela International Headache Society6.

d) Critérios de diagnóstico clínico do bruxismo do sonol que se baseia no relato do ranger dos dentes pelo paciente, somado a 
pelo menos um dos seguintes itens: a) desgaste anormal dos dentes; b) dor ou fadiga dos músculos da mastigação ao acordar; c) hipertrofia do músculo masseter na máxima contração voluntária. Resultado: A amostra foi composta por 286 indivíduos (84.3\% mulheres), com média de idade de 37.3 anos. Foi encontrada uma associação significativa entre DTM dolorosa, Migrânea a Cefaleia do Tipo Tensional (CTT). A magnitude da associação foi maior para a migrânea crônica (MC), seguida pela migrânea episódica (ME) e CTT episódica (CTTE) (Tabela 1). Em relação o BS, a associação foi significativa somente para MC (Tabela 2). Quando a amostra foi estratificada pela presença de BS e DTM dolorosa, somente a presença de $B S$ não aumentou o risco para as cefaleias primárias. $A$ presença de DTM dolorosa sem BS aumentou significativamente $O$ risco, em maior magnitude para MC, seguida pela ME e CTTE. A associação entre DTM dolorosa e BS aumentou significantemente o risco para MC, seguida pela ME e CTTE (Tabela 3). Discussão: Estudos que investigam a múltipla associação entre DTM dolorosa, BS e cefaleias primárias ainda são inexistentes. $O$ presente estudo encontrou uma associação significativa entre essas três entidades.

Tabela 1. Associação entre o diagnóstico de DTM dolorosa de acordo com o diagnóstico da cefaleia primária.

\begin{tabular}{|c|c|c|c|c|}
\hline & \multicolumn{2}{|c|}{ DTM Dolorosa } & \multirow{2}{*}{$\begin{array}{l}\text { Total } \\
\mathrm{n}(\%)\end{array}$} & \multirow{2}{*}{$\begin{array}{l}\text { Odds Ratio } \\
\text { (OR) 95\% } \\
\text { Intervalo de } \\
\text { Confiança (IC) }\end{array}$} \\
\hline & $\begin{array}{c}\text { Presente } \\
n(\%)\end{array}$ & $\begin{array}{c}\text { Ausente } \\
n(\%)\end{array}$ & & \\
\hline $\begin{array}{l}\text { Ausência de } \\
\text { Cefaleia }\end{array}$ & $\begin{array}{c}27 \\
(12.3)\end{array}$ & $\begin{array}{c}37 \\
(55.2)\end{array}$ & $\begin{array}{c}64 \\
(22.4)\end{array}$ & Referência \\
\hline $\begin{array}{l}\text { Migrânea } \\
\text { Episódica }\end{array}$ & $\begin{array}{c}92 \\
(83.6)\end{array}$ & $\begin{array}{c}18 \\
(16.4)\end{array}$ & $\begin{array}{l}110 \\
(100)\end{array}$ & $\begin{array}{c}7.0(3.45-14.22) \\
p<0.0001\end{array}$ \\
\hline $\begin{array}{l}\text { Cefaleia do } \\
\text { Tipo Tensional } \\
\text { Episódica }\end{array}$ & $\begin{array}{c}30 \\
(73.2)\end{array}$ & $\begin{array}{c}11 \\
(26.8)\end{array}$ & $\begin{array}{c}41 \\
(100)\end{array}$ & $\begin{array}{c}3.7(1.59-8.75) \\
p=0.0036\end{array}$ \\
\hline $\begin{array}{l}\text { Migrânea } \\
\text { Crônica }\end{array}$ & $\begin{array}{c}70 \\
(98.6)\end{array}$ & $\begin{array}{c}1 \\
(1.4)\end{array}$ & $\begin{array}{c}71 \\
(100)\end{array}$ & $\begin{array}{c}95.9(12.51-734.64) \\
p<0.0001\end{array}$ \\
\hline $\begin{array}{l}\text { Total } \\
(n \%)\end{array}$ & $\begin{array}{l}219 \\
(76.6)\end{array}$ & $\begin{array}{c}67 \\
(23.4)\end{array}$ & $\begin{array}{c}286 \\
(100.0)\end{array}$ & \\
\hline
\end{tabular}

Desde que essas condições são altamente prevalentes e apresentam um grande impacto na qualidade de vida dos indivíduos, nossos resultados contribuem para o conhecimento atual. Recentemente, alguns estudos demonstram uma relação de comorbidade entre DTM e cefaleias primárias. A hipótese mais plausível para essa é que impulsos nociceptivos dos músculos da mastigação e articulações temporomandibulares estimulam o núcleo caudal trigeminal, que é o conduto sensitivo tanto das dores orofaciais como das cefaleias, podendo aumentar o risco para o início de uma crise migranosa4. A respeito da associação entre BS e DTM, nossos resultados mostraram diferentes riscos, de acordo com o diagnóstico de cefaleia primária. Para migrânea, o BS poderia ser um fator de risco para DTM dolorosa e até mesmo estar colaborando com o processo de cronificação. Para a CTTE, o risco foi observado somente quando a DTM dolorosa e o BS estavam associados. As características clínicas de CTTE 6 são semelhantes às características da DTM associada ao SB. Há situações em que, possivelmente, essas características não são tão evidentes para permitir o diagnóstico diferencial. Além disso, a DTM associada ao BS e CTTE

Tabela 2. Associação entre diagnóstico de bruxismo do sono de acordo com o diagnóstico da cefaleia primária

\begin{tabular}{|c|c|c|c|c|}
\hline & \multicolumn{2}{|c|}{ Bruxismo do Sono } & \multirow{2}{*}{$\begin{array}{l}\text { Total } \\
\mathrm{n}(\%)\end{array}$} & \multirow[b]{2}{*}{ OR 95\% IC } \\
\hline & $\begin{array}{c}\text { Presente } \\
\mathrm{n}(\%)\end{array}$ & $\begin{array}{c}\text { Ausente } \\
\mathrm{n}(\%)\end{array}$ & & \\
\hline $\begin{array}{l}\text { Ausência de } \\
\text { Cefaleia }\end{array}$ & $\begin{array}{c}28 \\
(43.7)\end{array}$ & $\begin{array}{c}36 \\
(56.3)\end{array}$ & $\begin{array}{c}64 \\
(100.0)\end{array}$ & Reference \\
\hline $\begin{array}{l}\text { Migrânea } \\
\text { Episódica }\end{array}$ & $\begin{array}{c}64 \\
(58.2)\end{array}$ & $\begin{array}{c}46 \\
(41.8)\end{array}$ & $\begin{array}{c}110 \\
(100.0)\end{array}$ & $\begin{array}{c}1.8(0.96-3.33) \\
p=0.0927\end{array}$ \\
\hline $\begin{array}{l}\text { Cefaleia do } \\
\text { Tipo Tensional } \\
\text { Episódica }\end{array}$ & $\begin{array}{c}23 \\
(56.1)\end{array}$ & $\begin{array}{c}18 \\
(43.8)\end{array}$ & $\begin{array}{c}41 \\
(100.0)\end{array}$ & $\begin{array}{c}1.6(0.75-3.62) \\
p=0.3007\end{array}$ \\
\hline $\begin{array}{l}\text { Migrânea } \\
\text { Crônica }\end{array}$ & $\begin{array}{c}53 \\
(74.6)\end{array}$ & $\begin{array}{c}18 \\
(25.4)\end{array}$ & $\begin{array}{c}71 \\
(100.0)\end{array}$ & $\begin{array}{c}3.8(1.83-7.84) \\
p=0.0005\end{array}$ \\
\hline $\begin{array}{l}\text { Total } \\
(n \%)\end{array}$ & $\begin{array}{c}168 \\
(58.7)\end{array}$ & $\begin{array}{c}121 \\
(41.3)\end{array}$ & $\begin{array}{c}286 \\
(100.0)\end{array}$ & \\
\hline
\end{tabular}

Tabela 3. Associação entre o diagnóstico das cefaleias primárias de acordo com a frequência e a presença de Disfunção Temporomandibular e Bruxismo do Sono.

\begin{tabular}{|c|c|c|c|c|c|c|c|c|}
\hline & $\begin{array}{c}\text { Sem } \\
\text { Cefaleia } \\
n(\%)\end{array}$ & $\begin{array}{l}M E \\
n(\%)\end{array}$ & $\begin{array}{c}\text { OR } \\
95 \% \text { IC }\end{array}$ & $\begin{array}{c}\text { CTTE } \\
\mathrm{n}(\%)\end{array}$ & $\begin{array}{c}\text { OR } \\
95 \% \text { IC }\end{array}$ & $\begin{array}{l}M C \\
\mathrm{n}(\%)\end{array}$ & $\begin{array}{c}\text { OR } \\
95 \% \text { IC }\end{array}$ & $\begin{array}{l}\text { Total } \\
\mathrm{n}(\%)\end{array}$ \\
\hline - DTM -BS n(\%) & $23(35.9)$ & 15 (13.6) & Referência & $9(21.9)$ & Referência & $1(1.4)$ & Referência & $48(16.8)$ \\
\hline - DTM + BS n(\%) & $14(21.9)$ & $3(2.7)$ & $\begin{array}{c}0.3 \\
(0.08-1.34) \\
p=0.1330\end{array}$ & $2(5.0)$ & $\begin{array}{c}0.4 \\
(0.07-1.94) \\
p=0.3954\end{array}$ & $0(0.0)$ & $\begin{array}{c}0.5 \\
(0.02-14.18) \\
p=1\end{array}$ & $19(6.6)$ \\
\hline + DTM -BS n(\%) & $13(20.3)$ & $31(28.2)$ & $\begin{array}{c}3.7 \\
(1.46-9.16) \\
p=0.0094\end{array}$ & $9(21.9)$ & $\begin{array}{c}1.8 \\
(0.56-5.57) \\
p=0.4931\end{array}$ & $17(23.9)$ & $\begin{array}{c}30.1 \\
(3.58-252.81) \\
p<0.0001\end{array}$ & $70(24.5)$ \\
\hline + DTM + BS n(\%) & $14(21.9)$ & $61(55.5)$ & $\begin{array}{c}6.7 \\
(2.79-15.98) \\
p<0.0001\end{array}$ & $21(51.2)$ & $\begin{array}{c}3.8 \\
(1.38-10.69) \\
p=0.0176\end{array}$ & $53(74.7)$ & $\begin{array}{c}87.1 \\
(10.79-702.18) \\
p<0.0001\end{array}$ & $149(52.1)$ \\
\hline Total nl\% & $64(100.0)$ & $110(100.0)$ & & $41(100.0)$ & & $71(100.0)$ & & $286(100.0$ \\
\hline
\end{tabular}

ME = Migrânea Episódica; CTTE = Cefaleia do Tipo Tensional Episódica; MC= Migrânea Crônica; DTM= Disfunção Temporomandibular; $\mathrm{BS}=$ Bruxismo do Sono 
podem co-existir no mesmo paciente, e ambos poderiam ser diagnosticadas, dependendo da especialidade dos profissionais7. Portanto, é importante salientar que os critérios atuais de diagnóstico não permitem ao clínico diferenciar uma DTM iniciada por SB da CTTE. Isso exigiria uma revisão dos seus critérios de diagnóstico. É importante ressaltar que o presente estudo não permite que uma relação de causalidade seja estabelecida. Em conclusão, o estudo mostra que a associação BS-DTM dolorosa aumenta consideravelmente o risco para a ME e CTTE e, principalmente, para a MC. Por este motivo, recomendamos a interação entre neurologistas e dentistas na avaliação e gerenciamento de pacientes que sofrem de dor facial e cefaleias primárias.

\section{Referências}

1. American Academy of Sleep Medicine. International classification of sleep disorders: diagnostic and coding manual. 2nd ed. Westchester, Illinois: American Academy of Sleep Medicine; 2005
2. De Leeuw R. Orofacial pain: guidelines for assessment, diagnosis, and management. 4th ed. Chicago: Quintessence. 2008.

3. McNeill C. Management of temporomandibular disorders: concepts and controversies. J Prosthet Dent. 1997; 77: 510-22

4. Gonçalves DAG, Bigal ME, Jales LCF, Camparis CM, Speciali JG. Headache and symptoms of temporomandibular disorder: an epidemiological study. Headache. 2010; 50: 231-41.

5. Molina OF, Santos J Jr., Nelson SJ, Grossman E. Prevalence of modalities of headache and bruxism among patients with craniomandibular disorder. Cranio. 1997; 15: 314-25.

6. Subcomitê de Classificação Internacional das Cefaléias da Sociedade Internacional de Cefaléia. Classificação Internacional das Cefaléias. 2nd ed. Trad. Sociedade Brasileira de Cefaléia. São Paulo: Alaúde Editorial; 2006. 286 p.

7. Siqueira JTT. Disfunção temporomandibular: classificação e abordagem clínica. In: Siqueira JTT, Teixeira MJ. Dor orofacial: diagnóstico, terapêutica e qualidade de vida. Curitiba: Editora Maio; 2001. p. 374-404

Apoio financeiro: FAPESP - Auxilio Pesquisa/Processo: 06/00730-5

Protocolo de Aprovação do Comitê de Ética: Comitê de Ética em Pesquisa da Faculdade de Odontologia de Araraquara/UNESP (no do protocolo - 33/

\title{
DOR 13 - Influência da dor por disfunção temporomandibular e migrânea na presença de zumbido
}

\author{
Soares RCV'; Fernandes $\mathrm{G}^{2}$; Gonçalves $\mathrm{DAG}^{3}$; Franco $\mathrm{AL}^{4}$; Camparis $\mathrm{CM}^{5}$ \\ 'Aluna de Graduação; ${ }^{2,4}$ Aluna de Pós-Graduação; ${ }^{3}$ Professora Assistente; ${ }^{5}$ Professor Adjunto. Disciplina de Oclusão, \\ Desordens Temporomandibulares e Dores Orofaciais, Departamento de Materiais Odontológicos e Prótese, \\ Faculdade de Odontologia de Araraquara, UNESP, SP, Brasil
}

Introdução: Disfunção temporomandibular (DTM) é um termo coletivo que abrange problemas clínicos envolvendo a musculatura da mastigação, a articulação temporomandibular (ATM) e estruturas associadas (Okeson, 1996). A prevalência de zumbido em pacientes com DTM varia de 33 a $76 \%$, sendo maior do que a encontrada na população em geral, que é de 15\% (Lam, Lawrence e Tenebaum, 2001; Ahmad e Seidman, 2004). O zumbido pode ser definido como uma sensação de som percebida pelo indivíduo, independentemente de estímulo sonoro externo, devendo sempre ser considerado sintoma de alguma doença (Ahmad e Seidman, 2004). Atualmente, há poucas evidências sobre a relação fisiopatológica entre DTM e Zumbido. A hipótese mais aceita é que o sistema nervoso central, quando sensibilizado, estaria envolvido na geração e percepção do zumbido (Levine, 2003; Ahmad e Seidman, 2004). Além disso, tem sido demonstrada uma relação de comorbidade entre DTM e Migrânea, estando essa interação relacionada ao nervo trigêmeo, que é o conduto sensitivo tanto das cefaleias quanto das dores orofaciais. Sugere-se que essas são entidades distintas atuando uma sobre a outra como fator perpetuante e agravante (Gonçalves et al., 2010). Em relação ao zumbido, em uma série de casos, percebeu-se que pacientes com migrânea apresentam zumbido durante a crise migranosa (Volcy et al., 2005). Entretanto, ainda não há estudos sobre a relação entre zumbido, DTM e migrânea. Proposição: Baseado na literatura prévia, o objetivo do presente estudo é investigar uma possível associação entre DTM dolorosa, migrânea e zumbido, e estimar o risco de ocorrência de zumbido em pacientes com tais condições. Material e Método: Após o diagnóstico diferencial em relação a outras fontes de dor orofacial, o Research Diagnostic Criteria for Temporomandibular Disorders (RDC/TMD), foi usado para classificação da DTM. O RDC/TMD é um instrumento amplamente usado em pesquisa e é composto por um questionário e exame clínico que classifica a DTM em Eixo I (diagnósticos físicos) e Eixo II (grau de dor crônica, depressão, somatização e limitação da função mandibular). A presença de zumbido foi avaliada por meio de auto-relato. A migrânea foi diagnosticada e classificada, em episódica ou crônica, por meio de um questionário validado (Gonçalves et al., 2010) baseado na Classificação Internacional de Cefaléias-II. Os testes do $\chi^{2}$ e Odds Ratio (OR) com 95\% Intervalo de Confiança (IC) foram aplicados com nível de significância de 0,05. Resultado: A amostra foi composta por 245 indivíduos $(210$ mulheres e 35 homens), com média de idade de 37,15 anos (18 - 76 anos). Para investigar a relação entre a presença de zumbido com DTM dolorosa e migrânea crônica e episódica, a amostra foi estratificada de acordo com as tabelas abaixo. Na tabela 1, é possível observar que a DTM dolorosa aumentou o risco para a presença de zumbido $(O R=4,995 \%$ IC: 2,04-11,63; $p=0,0005)$. Da mesma forma

Tabela 1: Distribuição a amostra de acordo com a presença de DTM dolorosa e Zumbido

\begin{tabular}{|c|c|c|c|c|}
\hline & \multicolumn{2}{|c|}{ Zumbido } & \multirow{2}{*}{$\begin{array}{l}\text { Total } \\
\mathrm{n}(\%)\end{array}$} & \multirow[b]{2}{*}{ OR 95\% IC } \\
\hline & $\begin{array}{c}\text { Ausência } \\
n(\%)\end{array}$ & $\begin{array}{c}\text { Presença } \\
\mathrm{n}(\%)\end{array}$ & & \\
\hline $\begin{array}{l}\text { Sem DTM Dolorosa } \\
(\mathrm{n} \%)\end{array}$ & $\begin{array}{c}38 \\
(60.3)\end{array}$ & $\begin{array}{c}10 \\
(23.8)\end{array}$ & $\begin{array}{c}48 \\
(45.7)\end{array}$ & Referência \\
\hline $\begin{array}{l}\text { DTM Dolorosa } \\
(\mathrm{n} \%)\end{array}$ & $\begin{array}{c}25 \\
(39.7)\end{array}$ & $\begin{array}{c}32 \\
(76.2)\end{array}$ & $\begin{array}{c}57 \\
(54.3)\end{array}$ & $\begin{array}{c}4.9(2.04-11.63) \\
p=0,0005\end{array}$ \\
\hline $\begin{array}{l}\text { Total } \\
(\mathrm{n} \%)\end{array}$ & $\begin{array}{c}63 \\
(100.0)\end{array}$ & $\begin{array}{c}42 \\
(100.0)\end{array}$ & $\begin{array}{c}105 \\
(100.0)\end{array}$ & \\
\hline
\end{tabular}


Tabela 2: Distribuição a amostra de acordo com a presença de migrânea episódica e crônica e Zumbido

\begin{tabular}{|c|c|c|c|c|}
\hline & \multicolumn{2}{|c|}{$\begin{array}{l}\text { Zumbido auto- } \\
\text { relatado }\end{array}$} & \multirow{2}{*}{$\begin{array}{l}\text { Total } \\
n(\%)\end{array}$} & \multirow{2}{*}{ OR 95\% IC } \\
\hline & $\begin{array}{c}\text { Ausência } \\
\mathrm{n}(\%)\end{array}$ & $\begin{array}{c}\text { Presença } \\
\mathrm{n}(\%)\end{array}$ & & \\
\hline $\begin{array}{c}\text { Sem Cefáleia } \\
(n \%)\end{array}$ & $\begin{array}{c}20 \\
(13.6)\end{array}$ & $\begin{array}{c}44 \\
(44.9)\end{array}$ & $\begin{array}{c}64 \\
(26.1)\end{array}$ & Referência \\
\hline $\begin{array}{l}\text { Migrânea Episódica } \\
\text { (n\%) }\end{array}$ & $\begin{array}{c}70 \\
(47.6)\end{array}$ & $\begin{array}{c}40 \\
(40.8)\end{array}$ & $\begin{array}{c}110 \\
(44.9)\end{array}$ & $\begin{array}{c}3.9(1.99-7.42) \\
p<0.0001\end{array}$ \\
\hline $\begin{array}{c}\text { Migrânea Crônica } \\
\text { (n\%) }\end{array}$ & $\begin{array}{c}57 \\
(38.8)\end{array}$ & $\begin{array}{c}14 \\
(14.3)\end{array}$ & $\begin{array}{c}71 \\
(29.9)\end{array}$ & $\begin{array}{c}18.9(4.07-19.70) \\
p<0.0001\end{array}$ \\
\hline $\begin{array}{l}\text { Total } \\
(n \%)\end{array}$ & $\begin{array}{c}147 \\
(100.0)\end{array}$ & $\begin{array}{c}98 \\
(100.0)\end{array}$ & $\begin{array}{c}245 \\
(100.0)\end{array}$ & \\
\hline
\end{tabular}

Tabela 3: Associação entre auto-relato de zumbido de acordo com a presença de DTM dolorosa e migrânea crônica e episódica

\begin{tabular}{|c|c|c|c|c|}
\hline & \\
\hline & $\begin{array}{c}\text { Zumbido } \\
\text { ausente } \\
N(\%)\end{array}$ & $\begin{array}{c}\text { Zumbido } \\
\text { Presente } \\
N(\%)\end{array}$ & $\begin{array}{l}\text { Total } \\
N(\%)\end{array}$ & $\begin{array}{l}\text { OR }(95 \% \text { IC) } \\
\text { Valor de p }\end{array}$ \\
\hline - DTM - M & $32(32.7)$ & $6(4.0)$ & $38(15.5)$ & Referência \\
\hline+ DTM - M & $12(12.2)$ & $14(9.5)$ & $26(10.6)$ & $\begin{array}{c}6.22(1.94-19.93) \\
p=0.0022\end{array}$ \\
\hline - DTM + ME & $11(11.2)$ & $7(4.8)$ & $18(7.3)$ & $\begin{array}{c}3.39(0.93-12.30) \\
p=0.0889\end{array}$ \\
\hline - DTM + MC & $1(1.0)$ & $0(0.0)$ & $1(0.4)$ & $\begin{array}{c}1.66(0.06-45.65) \\
p=1.0\end{array}$ \\
\hline$+\mathrm{DTM}+\mathrm{ME}$ & $29(29.6)$ & $63(42.9)$ & $92(37.6)$ & $\begin{array}{c}11.58(4.36-30.77) \\
p<0.0001\end{array}$ \\
\hline$+\mathrm{DTM}+\mathrm{MC}$ & $13(13.3)$ & 57 (38.8) & $70(28.6)$ & $\begin{array}{c}23.38(8.10-67.50) \\
p<0.0001\end{array}$ \\
\hline Total & $98(100.0)$ & $\begin{array}{c}147 \\
(100.0)\end{array}$ & $245(100.0)$ & \\
\hline
\end{tabular}

DTM = Disfunção Temporomandibular; $M=$ Migrânea; $M E=$ Migrânea Episódica; $M C=$ Migrânea Crônica.

também foi encontrado um risco aumentado para o zumbido na presença de migrânea $(p<0,0001)$, sendo em maior magnitude para a migrânea crônica $(18,9 ; 4,07-19,70)$, que para migrânea episódica $(3,9 ; 1,99-7,42)$ (Tabela 2). A amostra foi então agrupada de acordo com a presença simultânea de DTM dolorosa, migrânea crônica e episódica. A associação de DTM dolorosa com migrânea crônica e episódica aumenta o risco para zumbido quando comparado com a presença isolada das mesmas $(p<0,0001)$, sendo em maior magnitude na presença de migrânea crônica $(23,38 ; 8,10-67,50)$, seguida pela migrânea episódica (1 1,58; 4,36-30,77) (tabela3). Discussão: $\bigcirc$ presente estudo demonstrou associação entre DTM dolorosa, migrânea e zumbido, sendo essas condições altamente prevalentes e de grande impacto na qualidade de vida desses pacientes, esse estudo contribui para os conhecimentos atuais. A presença de DTM dolorosa aumentou o risco para o zumbido. Atualmente, a hipótese mais aceita para explicar essa associação é baseada na sensibilização central. Os núcleos grácil e cuneiforme presentes na via somatossensorial apresentam uma projeção excitatória para o núcleo coclear dorsal das vias auditivas. Esses núcleos, uma vez sensibilizados, levariam estímulos dolorosos gerados na via somatossensorial ao córtex auditivo que, por sua vez, os interpretaria como zumbido (Levine, 2003; Sanchez et al., 2003). Além disso, a presença de migrânea aumentou o risco para o zumbido, sendo em maior magnitude para a migrânea crônica. Para explicar esses resultados, sugerimos um funcionamento anormal do córtex auditivo devido ao processo de sensibilização central, já citado anteriormente, gerado durante as crises migranosas (Volcy et al., 2005). $\bigcirc$ risco apresenta-se aumentado quando a migrânea e a DTM estão associadas, pois essas quando somadas poderiam levar a uma maior sensibilização do sistema nervoso central e consequentemente a um funcionamento anormal do córtex auditivo. Conclusão: A DTM dolorosa, migrânea crônica e episódica podem ser um fator de risco para o zumbido, sendo esse risco aumentado quando essas duas condições coexistem no mesmo paciente. Por isso, sugerimos um tratamento interdisciplinar na avaliação e gerenciamento de pacientes que sofrem de DTM, Migrânea e Zumbido.

Referências

1. Ahmad N, Seidman M. Tinnitus in Older Adult: Epidemiology, Pathophysiology and Treatment Options. Drugs Aging. 2004; 21:297-305.

2. Gonçalves DAG, Bigal ME, Jales LCF, Camparis CM, Speciali JG. Headache and symptoms of temporomandibular disorder: an epidemiological study. Headache. 2010; 50: 231-41.

3. Lam DK, Lawrence HP, Tenenbaum HC. Aural symptoms in temporomandibular disorder patients attending a craniofacial pain. J Orofac Pain. $2001 ; 15: 146-57$.

4. Levine RA. Somatic (Craniocervical) tinnitus and the dorsal cochlear nucleus hypothesis. Am J Otolaryngol. 1999; 20: 351-62.

5. Okeson JP. Orofacial pain: guidelines for assessment, diagnosis, and management. 3th ed. Chicago: Quintessence; 1996.

6. Sanchez TG, Brandão AL, Lima AS, Lorenzi MC, Bento RF. Reprodutibilidade do teste de modulação somática do zumbido e resultados após treinamento com contrações musculares repetidas. Arq Int Otorrinolaringol. 2003; 7: 237.

7. Volcy M, Sheftell FD, Tepper SJ, Rapoport AM, Bigal ME. Tinnitus in Migraine: An allodynic symptom secondary to abnormal cortical functioning? Headache.2005; 45:1083-7.

Apoio financeiro: FAPESP - Auxilio Pesquisa/Processo: 06/00730-5

Protocolo de Aprovação do Comitê de Ética: Comitê de Ética em Pesquisa da Faculdade de Odontologia de Araraquara/UNESP (no do protocolo - 33/06) 